\title{
Robust Controller Synthesis in Timed Automata
}

Ocan Sankur, Patricia Bouyer, Nicolas Markey,

Pierre-Alain Reynier

April 2013

Research report LSV-13-08 (Version 1)

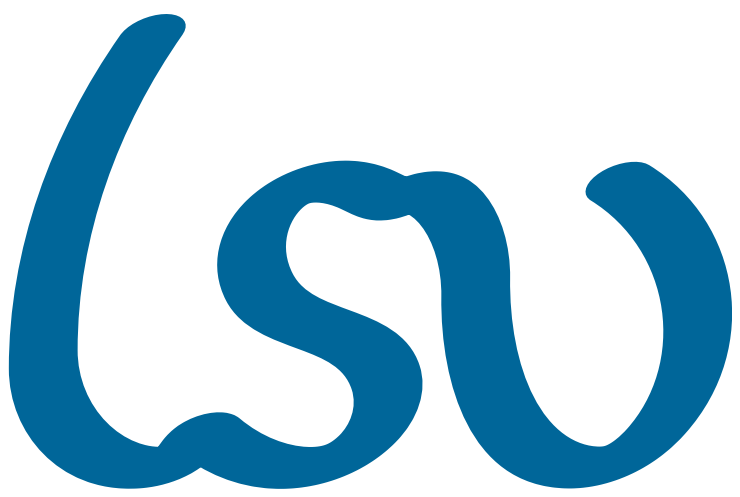

Laboratoire Spécification \& Vérification

École Normale Supérieure de Cachan

61, avenue du Président Wilson

94235 Cachan Cedex France 



\title{
Robust Controller Synthesis in Timed Automata
}

\author{
Ocan Sankur ${ }^{1}$, Patricia Bouyer ${ }^{1}$, Nicolas Markey ${ }^{1}$, Pierre-Alain Reynier ${ }^{2}$ \\ 1 LSV, ENS Cachan \& CNRS, France \\ ${ }^{2}$ LIF, Aix-Marseille Université \& CNRS, France
}

\begin{abstract}
We consider the fundamental problem of Büchi acceptance in timed automata in a robust setting. The problem is formalised in terms of controller synthesis: timed automata are equipped with a parametrised game-based semantics that models the possible perturbations of the decisions taken by the controller. We characterise timed automata that are robustly controllable for some parameter, with a simple graph theoretic condition, by showing the equivalence with the existence of an aperiodic lasso that satisfies the winning condition (aperiodicity was defined and used earlier in different contexts to characterise convergence phenomena in timed automata). We then show decidability and PSPACE-completeness of our problem.
\end{abstract}

\section{Introduction}

Timed automata [AD94] are a timed extension of finite-state automata, providing an automata-theoretic framework to design, model, verify and synthesise systems with timing constraints. However, the semantics of timed automata is an idealisation of real timed systems; it assumes, for instance, perfect clocks for arbitrarily precise time measures, and instantaneous actions. Thus, properties proven on timed automata may not hold in a real implementation, and similarly, a synthesised controller may not be realisable on a real hardware. This problem has been addressed in several works in the literature, where the goal is to define a convenient notion of robustness, so as to define a realistic semantics for timed automata, and also make sure that the verified (or synthesised) behaviour remains correct in presence of small perturbations.

In this work, we consider the fundamental problem of Büchi acceptance of a given timed automaton in a robust setting. Our goal is to distinguish timed automata where a Büchi condition can be satisfied even when the chosen delays are systematically perturbed by an adversary by a bounded parametrised amount. In fact, it has been observed that some timed automata require choosing time delays with infinite precision in order to realise some behaviours. Apart from well-known Zeno behaviours, [CHR02] shows such a convergence phenomenon where an infinite run requires increasing precision at each step. These unrealisable behaviours are discarded in such an adversarial robust setting. Thus, we formalize the problem in a game-theoretic setting. Our objective is to synthesise controllers that are robust while discarding unrealisable behaviours.

More precisely, to define robustness, we equip timed automata with the following game semantics between two players ([CHP11]): Controller with a 
given Büchi objective, and Perturbator with the complementary objective. The semantics is a turn-based game parametrised by $\delta>0$. At each step, Controller chooses an edge, and $d>\delta$, such that the guard of the edge is satisfied after any delay $d^{\prime} \in[d-\delta, d+\delta]$. Then, the edge is taken after a delay $d^{\prime} \in[d-\delta, d+\delta]$ chosen by Perturbator. Timed games with parity conditions were studied in [CHP11] for a fixed known parameter $\delta>0$, and for strictly positive delays with no lower bound. In fact, in this case, one can encode this semantics as a usual timed game, and rely on existing techniques for solving timed games. For an unknown parameter $\delta>0$, the problem is more complicated, and was left as a challenging open problem in [CHP11].

Our main result is the following: we show that deciding the existence of $\delta>0$, and of a strategy for Controller in the perturbation game so as to ensure infinite runs satisfying a given Büchi condition is PSPACE-complete, thus no harder than in the exact setting [AD94]. We characterise robust timed automata, i.e., those in which Controller has a winning strategy, by showing that Controller can win precisely when the timed automaton has an accepting aperiodic lasso. Aperiodicity [Sta12] is a variant of forgetfulness introduced in [BA11] in a different context, to study the entropy of timed languages. Our characterisation confirms the suggestion of [BA11] that this notion could be significant in the study of robustness. Our results rely on the non-trivial combination of various techniques used for studying timed automata: forgetful and aperiodic cycles as considered in [BA11,Sta12], the metrics of [GHJ97], shrinking techniques [SBM11,BMS12] and reachability relations of [Pur00]. Last, our proof provides a symbolic representation of Controller's strategy which could be amenable to implementation.

Related works. A similar game semantics was considered in [BMS12], but the winning objectives considered are only reachability. An important consequence is that convergence phenomena, and unrealisable strategies are not an issue, since one essentially only considers finite paths. In this paper, we thus need new proof techniques to deal with convergence. In addition, the semantics considered in [BMS12] is less restrictive for Controller: he only needs to suggest delays after which the guard of the chosen edge is satisfied. Hence, the guard may not be satisfied after a perturbation. The emphasis in the resulting semantics is therefore on the newly appearing behaviours. Algorithmically, the semantics of [BMS12] gives rise to more complex problems: reachability is already EXPTIME-complete, whereas we are able to treat richer Büchi objectives in PSPACE in this paper. From a designer's perspective, we believe that both semantics are meaningful in different modelling assumptions. The present semantics is interesting if lower and upper bounds on events, e.g task execution times, appear naturally in the model, and need be respected strictly. On the other hand, in other applications, the semantics of [BMS12] allows to model with equality constraints, and then synthesise controllers taking into account additional behaviour due to perturbations.

A related line of work is that of [Pur00,DDR05,DDMR08], which consists in modeling imprecisions by enlarging all clock constraints of the automaton by some parameter $\delta$, that is, transforming each constraint of the form $x \in[a, b]$ into $x \in[a-\delta, b+\delta]$. The dual notion of shrinking was considered in [SBM11] in 
order to study whether any significant behaviour is lost when guards are shrunk. Both approaches are interested in model-checking, and the robustness condition is defined on the global behaviour of the enlarged or shrunk timed automaton. This does not capture the system's ability to adapt to perturbations that were observed earlier in a given run. In contrast, the game semantics endows Controller with a strategy against perturbations.

Among other robustness notions, [GHJ97] defines the tube semantics using a topology on timed automata runs. Our semantics is not directly related as we have a game semantics and a concrete parameter $\delta$. However we use some results from [GHJ97] in our proofs.

\section{Timed Automata and Robust Safety}

Given a finite set of clocks $\mathcal{C}$, we call valuations the elements of $\mathbb{R}_{>0}^{\mathcal{C}}$. For a subset $R \subseteq \mathcal{C}$ and a valuation $\nu, \nu[R \leftarrow 0]$ is the valuation defined by $\nu[R \leftarrow 0](x)=\nu(x)$ for $x \in \mathcal{C} \backslash R$ and $\nu[R \leftarrow 0](x)=0$ for $x \in R$. Given $d \in \mathbb{R}_{\geq 0}$ and a valuation $\nu$, the valuation $\nu+d$ is defined by $(\nu+d)(x)=\nu(x)+d$ for all $x \in \mathcal{C}$. We extend these operations to sets of valuations in the obvious way. We write $\mathbf{0}$ for the valuation that assigns 0 to every clock.

An atomic clock constraint is a formula of the form $k \preceq x \preceq^{\prime} l$ or $k \preceq x-y \preceq^{\prime} l$ where $x, y \in \mathcal{C}, k, l \in \mathbb{Z} \cup\{-\infty, \infty\}$ and $\preceq, \preceq^{\prime} \in\{<, \leq\}$. A guard is a conjunction of atomic clock constraints. A valuation $\nu$ satisfies a guard $g$, denoted $\nu \models g$, if all constraints are satisfied when each $x \in \mathcal{C}$ is replaced with $\nu(x)$. We write $\Phi_{\mathcal{C}}$ for the set of guards built on $\mathcal{C}$.

A timed automaton $\mathcal{A}$ is a tuple $\left(\mathcal{L}, \mathcal{C}, \ell_{0}, E\right)$, where $\mathcal{L}$ is a finite set of locations, $\mathcal{C}$ is a finite set of clocks, $E \subseteq \mathcal{L} \times \Phi_{\mathcal{C}} \times 2^{\mathcal{C}} \times \mathcal{L}$ is a set of edges, and $\ell_{0} \in \mathcal{L}$ is the initial location. An edge $e=\left(\ell, g, R, \ell^{\prime}\right)$ is also written as $\ell \stackrel{g, R}{\longrightarrow} \ell^{\prime}$.

The set of possible behaviours of a timed automaton can be described by the set of its runs, as follows. A run of $\mathcal{A}$ is a sequence $q_{1} e_{1} q_{2} e_{2} \ldots$ where $q_{i} \in \mathcal{L} \times \mathbb{R}_{\geq 0}^{\mathcal{C}}$, and writing $q_{i}=(\ell, \nu)$, either $e_{i} \in \mathbb{R}_{>0}$, in which case $q_{i+1}=\left(\ell, \nu+e_{i}\right)$, or $e_{i}=\left(\ell, g, R, \ell^{\prime}\right) \in E$, in which case $q_{i+1}=\left(\ell^{\prime}, \nu[R \leftarrow 0]\right)$. We denote by $\operatorname{state}_{i}(\rho)$ the $i$-th state of any run $\rho$, by first $(\rho)$ its first state, and, if $\rho$ is finite, last $(\rho)$ denotes the last state of $\rho$.

In order to define perturbations, and to capture the reactivity of a controller to these, we define the following robust game semantics, defined in [CHP11] (see also [BMS12] for a variant). Intuitively, the robust semantics of a timed automaton is a two-player game parametrised by $\delta>0$, where Player 1, also called Controller, chooses a delay $d>\delta$ and an edge whose guard is satisfied after any delay in the set $d+[-\delta, \delta]$. Then, Player 2, also called Perturbator, chooses an actual delay $d^{\prime} \in d+[-\delta, \delta]$ after which the edge is taken. Hence, Controller is required to always suggest delays that satisfy the guards whatever the perturbations are.

Formally, given a timed automaton $\mathcal{A}=\left(\mathcal{L}, \mathcal{C}, \ell_{0}, E\right)$ and $\delta>0$, we define the perturbation game of $\mathcal{A}$ w.r.t. $\delta$ as a two-player turn-based game $\mathcal{G}_{\delta}(\mathcal{A})$ between players Controller and Perturbator. The state space of $\mathcal{G}_{\delta}(\mathcal{A})$ is partitioned into $V_{C} \cup V_{P}$ where $V_{C}=\mathcal{L} \times \mathbb{R}_{\geq 0}^{\mathcal{C}}$ belong to Controller, and $V_{P}=\mathcal{L} \times \mathbb{R}_{\geq 0}^{\mathcal{C}} \times \mathbb{R}_{\geq 0} \times E$ belong to Perturbator. The initial state is $\left(\ell_{0}, \mathbf{0}\right) \in V_{C}$. The transitions are defined 


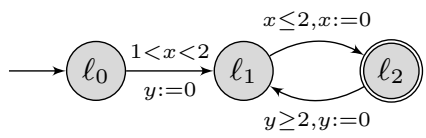

(a)

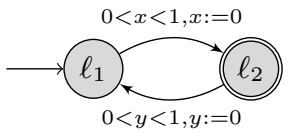

(b)

Fig. 1. On the left, a timed automaton from [Pur00] that is not robustly controllable for the Büchi objective $\left\{\ell_{2}\right\}$. In fact, Perturbator can enforce that the value of $x$ be increased by $\delta$ at each arrival at $\ell_{1}$, thus blocking the run eventually. On the right, the timed automaton (from [BA11]) is robustly controllable for the Büchi objective $\left\{\ell_{2}\right\}$. In fact, perturbations at a given transition do not affect the rest of the run; they are forgotten.

as follows: from any state $(\ell, \nu) \in V_{C}$, there is a transition to $(\ell, \nu, d, e) \in V_{P}$ whenever $d>\delta, e=\left(\ell, g, R, \ell^{\prime}\right)$ is an edge such that $\nu+d+\varepsilon=g$ for all $\varepsilon \in[-\delta, \delta]$. Then, from any such state $(\ell, \nu, d, e) \in V_{P}$, there is a transition to $\left(\ell^{\prime},(\nu+d+\varepsilon)[R \leftarrow 0]\right) \in V_{C}$, for any $\varepsilon \in[-\delta, \delta]$. A pair of states of $V_{C} \cup V_{P}$ is said to be consecutive if there is a transition between them. A play of $\mathcal{G}_{\delta}(\mathcal{A})$ is a finite or infinite sequence $q_{1} e_{1} q_{2} e_{2} \ldots$ of states and transitions of $\mathcal{G}_{\delta}(\mathcal{A})$, with $q_{1}=\left(\ell_{0}, \mathbf{0}\right)$, where $e_{i}$ is a transition from $q_{i}$ to $q_{i+1}$. It is said to be maximal if it is infinite or cannot be extended. A strategy for Controller is a function that assigns to every non-maximal play ending in some $(\ell, \nu) \in V_{C}$, a pair $(d, e)$ where $d>\delta$ and $e$ is an edge such that there is a transition from $(\ell, \nu)$ to $(\ell, \nu, d, e)$. A strategy for Perturbator is a function that assigns, to every play ending in $(\ell, \nu, d, e)$, a state $\left(\ell^{\prime}, \nu^{\prime}\right)$ such that there is a transition from the former to the latter state. A play $\rho$ is compatible with a strategy $f$ if for every prefix $\rho^{\prime}$ of $\rho$ ending in $V_{C}$, the next transition along $\rho$ after $\rho^{\prime}$ is given by $f$. We define similarly compatibility for Perturbator's strategies. A play naturally gives rise to a unique run, where the states are in $V_{C}$, the delays are those chosen by Perturbator, and the edges are chosen by Controller.

Given $\delta>0$, and a pair of strategies $f, g$, respectively for Controller and Perturbator we let Outcome ${ }_{\mathcal{A}}^{\delta}(f, g)$ denote the unique maximal run that is compatible with both strategies. We also define Outcome ${ }_{\mathcal{A}}^{\delta}(f, \cdot)\left(\operatorname{resp}\right.$. Outcome $\left.{ }_{\mathcal{A}}^{\delta}(\cdot, g)\right)$ as the set of all maximal runs compatible with $f$ (resp. with $g$ ). A Büchi objective is a subset of the locations of $\mathcal{A}$. Controller's strategy $f$ is winning for a Büchi objective $B$, if all runs of Outcome ${ }_{\mathcal{A}}^{\delta}(f, \cdot)$ are infinite and visit infinitely often some location of $B$. The parametrised robust controller synthesis problem asks, given a timed automaton $\mathcal{A}$ and a Büchi objective $B$, whether there exists $\delta>0$ such that Controller has a winning strategy in $\mathcal{G}_{\delta}(\mathcal{A})$ for the objective $B$. Note that these games are determined since for each $\delta>0$, the semantics is a timed game.

Figure 1 shows examples of controllable and uncontrollable timed automata, in our sense. The main result of this paper is the following.

Theorem 1. Parametrised robust controller synthesis is PSPACE-complete for Büchi objectives. 
The next section introduces several notions we need to state our main lemma (Lemma 3), which characterises timed automata that are robustly controllable, based on the nature of the lassos of the region automata.

\section{Regions, Orbit Graphs, Topology}

Regions and Region Automata. We assume that the clocks are bounded above by a known constant in all timed automata we consider. Fix a timed automaton $\mathcal{A}=\left(\mathcal{L}, \mathcal{C}, \ell_{0}, \mathcal{E}\right)$. We define regions as in [AD94], as subsets of $\mathbb{R}_{\geq 0}^{\mathcal{C}}$. Any region $r$ is defined by fixing the integer parts of the clocks, and giving a partition $X_{0}, X_{1}, \ldots, X_{m}$ of the clocks, ordered according to their fractional values: for any $\nu \in r, 0=\operatorname{frac}\left(\nu\left(x_{0}\right)\right)<\operatorname{frac}\left(\nu\left(x_{1}\right)\right)<\ldots<\operatorname{frac}\left(\nu\left(x_{m}\right)\right)$ for any $x_{0} \in X_{0}, \ldots, x_{m} \in X_{m}$, and $\operatorname{frac}(\nu(x))=\operatorname{frac}(\nu(y))$ for any $x, y \in X_{i}$. Here, $X_{i} \neq \emptyset$ for all $1 \leq i \leq m$ but $X_{0}$ might be empty. For any valuation $\nu$, let $[\nu]$ denote the region to which $\nu$ belongs.

We define the region automaton $\mathcal{R}(\mathcal{A})$ as a finite automaton whose states are pairs $(\ell, r)$ where $\ell \in \mathcal{L}$ and $r$ is a region. There is a transition $(\ell, r) \stackrel{\Delta}{\rightarrow}(\ell, s)$ if there exist $\nu \in r, \nu^{\prime} \in s$ and $d>0$ such that $\nu^{\prime}=\nu+d$. There is a transition $(\ell, r) \stackrel{e}{\rightarrow}\left(\ell^{\prime}, s\right)$ where $e=\left(\ell, g, R, \ell^{\prime}\right)$ if $r \models g$ and $r[R \leftarrow 0]=s$. We write the paths of the region automaton as $\pi=q_{1} e_{1} q_{2} e_{2} \ldots q_{n}$ where each $q_{i}$ is a state, and $e_{i} \in E \cup\{\Delta\}$, such that $q_{i} \stackrel{e_{i}}{\rightarrow} q_{i+1}$ for all $1 \leq i \leq n-1$. We also write $\operatorname{first}(\pi)=q_{1}$, last $(\pi)=q_{n}$, state $_{i}(\pi)=q_{i}$, and $\operatorname{trans}_{i}(\pi)=e_{i}$. The length of the path is $n$, and is denoted by $|\pi|$. We denote the subpath of $\pi$ between states of indices $i$ and $j$ by $\pi_{i \ldots j}$. Given a run $\rho$ of $\mathcal{A}$, its projection on regions is the path $\pi$ in the region automaton s.t. state $_{i}(\rho) \in$ state $_{i}(\pi)$ for all $1 \leq i \leq n$, and either $\operatorname{trans}_{i}(\rho)=\operatorname{trans}_{i}(\pi)$ or $\operatorname{trans}_{i}(\pi)=\Delta$ and $\operatorname{trans}_{i}(\rho) \in \mathbb{R}_{\geq 0}$. In this case, we write $\operatorname{first}(\rho) \stackrel{\pi}{\rightarrow} \operatorname{last}(\rho)$ (and say that $\rho$ is along $\pi$ ). A lasso is a path $\pi_{0} \pi_{1}$ where $\pi_{1}$ is a cycle, i.e. first $\left(\pi_{1}\right)=\operatorname{last}\left(\pi_{1}\right)$. A cycle of $\mathcal{R}(\mathcal{A})$ is a progress cycle if it resets all clocks at least once [Pur00].

A region $r$ is said to be non-punctual if it contains some $\nu \in r$ such that $\nu+[-\varepsilon, \varepsilon] \subseteq r$ for some $\varepsilon>0$. It is said punctual otherwise. By extension, we say that $(\ell, r)$ is non-punctual if $r$ is. A path $\pi=q_{1} e_{1} q_{2} e_{2} \ldots q_{n}$ is non-punctual if whenever $e_{i}=\Delta, q_{i+1}$ is non-punctual.

Vertices and Orbit Graphs. A vertex of a region $r$ is any point of $\bar{r} \cap \mathbb{N}^{\mathcal{C}}$, where $\bar{r}$ denotes the topological closure of $r$. For any region $r$, and any clock $x$, let us denote by $r_{x, 0}$ the upper bound (by $-r_{0, x}$ the lower bound) on clock $x$ inside region $r$. Then, a vertex $v$ of $r$ is defined by the choice of an index $1 \leq i \leq m$ such that for all $x \in X_{1}, \ldots, X_{i}$, we have $v(x)=-r_{0, x}$ and for all $x \in X_{i+1}, \ldots, X_{m}$, we have $v(x)=r_{x, 0}$. Hence, any region has at most $|\mathcal{C}|+1$ vertices (See e.g. [DDMR08]). We denote by $\inf (r)($ resp. $\sup (r))$ the vertex of $r$ where all clocks are equal to their lower bounds (resp. upper bounds). Let $\mathcal{V}(r)$ denote the set of vertices of $r$. We also extend this definition to $\mathcal{V}((\ell, r))=\mathcal{V}(r)$. Note the following easy properties of regions. Any region $r$ has at most one vertex $v \in \mathcal{V}(r)$ such that both $v$ and $v+1$ belong to $\mathcal{V}(r)$. If these exist, then $v=\inf (r)$ and $v+1=\sup (r)$. 
Moreover, $\sup (r)=\inf (r)+1$ if, and only if $r$ is non-punctual. It has been shown that all valuations in $r$ are convex combinations of $\mathcal{V}(r)$ [Pur00].

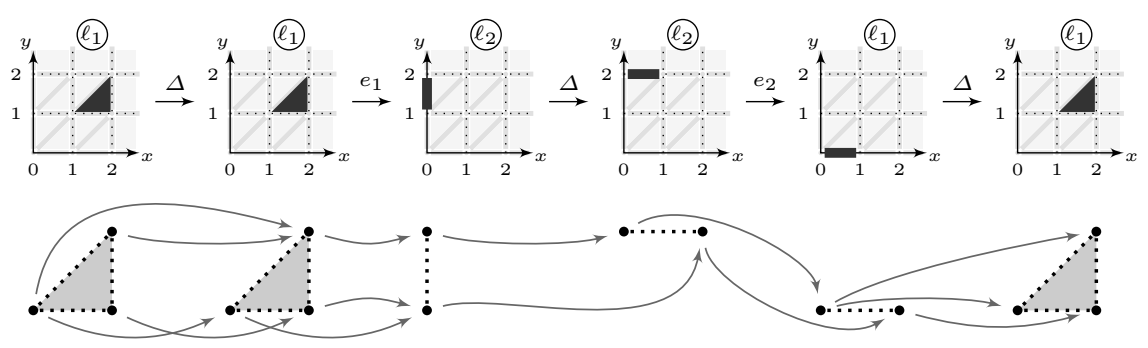

Fig. 2. The orbit graph of a (cyclic) path in the region automaton of the automaton of Fig. 1(a).

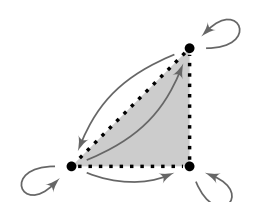

Fig. 3. The folded orbit graph of the (non-forgetful) cycle of Fig. 2.

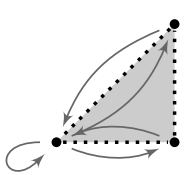

Fig. 4. The folded orbit graph of a forgetful cycle.

With any path $\pi$ of the region automaton, we associate a $|\pi|$-partite labelled graph $\gamma(\pi)$ called the orbit graph of $\pi$ [Pur00]. Intuitively, the orbit graph of a path gives the reachability relation between the vertices of the regions visited along the path. Formally, for a transition $\tau=q_{1} e_{1} q_{2}$, its orbit graph $\gamma(\tau)=\left(V_{1} \cup V_{2}, f_{G}, E\right)$ is a bipartite graph where $V_{1}=\{(1, v)\}_{v \in \mathcal{V}\left(q_{1}\right)}$, and $V_{2}=\{(2, v)\}_{v \in \mathcal{V}\left(q_{2}\right)}$. For any $((1, u),(2, v)) \in V_{1} \times V_{2}$, we have an edge $((1, u),(2, v)) \in E$, if, and only if $u \stackrel{\overline{e_{1}}}{\longrightarrow} v$, where $\overline{e_{1}}=\Delta$ if $e_{1}=\Delta$, and otherwise $\overline{e_{1}}$ is obtained by replacing the guard by its closed counterpart. Note that each vertex has at least one successor through $\overline{e_{1}}$ [AD94]. The labelling function $f_{G}$ maps each $i$ to $q_{i}$; we also extend to nodes of $G$ by $f_{G}((i, v))=f_{G}(i)$. In order to extend $\gamma(\cdot)$ to paths, we use a composition operator $\oplus$ between orbit graphs, defined as follows. If $G=\left(V_{1} \cup \ldots \cup V_{n}, f_{G}, E\right)$ and $G^{\prime}=\left(V_{1}^{\prime} \cup \ldots \cup V_{m}^{\prime}, f_{G^{\prime}}, E^{\prime}\right)$ denote two orbit graphs, then $G \oplus G^{\prime}$ is defined if, and only if, $f_{G}(n)=f_{G^{\prime}}(1)$, that is, when the path defining the former graph ends in the first state of the path defining the latter graph. In this case, the graph $G^{\prime \prime}=G \oplus G^{\prime}=\left(V_{1}^{\prime \prime} \cup \ldots V_{n+m-1}^{\prime \prime}, f_{G^{\prime \prime}}, E^{\prime \prime}\right)$ is defined by taking the disjoint union of $G$ and $G^{\prime}$, merging each node $(n, v)$ of $V_{n}$ with the node $(1, v)$ of $V_{1}^{\prime}$, and renaming any node $(i, v) \in V_{i}^{\prime}$ by $(i+n-1, v)$, so that we get a $(n+m-1)$-partite graph. Formally, we let $V_{i}=V_{i}^{\prime \prime}$ for all $1 \leq i \leq n$, and the subgraph of $G^{\prime \prime}$ induced on these nodes is equal to $G$. For any $n+1 \leq i \leq n+m-1$, we have $V_{i}^{\prime \prime}=\{(i, v)\}_{(i-n+1, v) \in V_{i-n+1}^{\prime}}$, and there is an edge $((i, v),(i+1, w)) \in E^{\prime \prime}$ if, and only if, $((i-n+1, v),(i-n, w)) \in E^{\prime}$. Now, we extend $\gamma(\cdot)$ to paths by induction, as follows. Consider any path $\pi=q_{1} e_{1} \ldots q_{n-1} e_{n-1} q_{n}$, and let $G=\left(V_{1} \cup \ldots \cup V_{n-1}, f_{G}, E\right)$ be the $(n-1)$ - 
partite graph $\gamma\left(q_{1} e_{1} \ldots q_{n-1}\right)$, given by induction. Let $G^{\prime}=\left(U \cup U^{\prime}, f_{G^{\prime}}, E^{\prime}\right)$ denote the bipartite graph of $q_{n-1} e_{n-1} q_{n}$. Then, we let $\gamma(\pi)=G \oplus G^{\prime}$. For any node $(i, v)$ of $\gamma(\pi)$, let $\operatorname{Succ}((i, v))$ denote the set of nodes $(i+1, w)$ with $((i, v),(i+1, w))$ is an edge. We also extend $\operatorname{Succ}(\cdot)$ to sets of nodes. Fig. 2 displays a path in the region automaton of the automaton depicted on Fig. 1(a) together with its orbit graph. Note that delays of duration zero are allowed when defining orbit graphs.

We define the folded orbit graph $\Gamma(\pi)$ for any path $\pi$ that is not a cycle, as a bipartite graph on node set $\{1\} \times \mathcal{V}($ first $(\pi)) \cup\{2\} \times \mathcal{V}($ last $(\pi))$. There is an edge $((1, v),(2, w))$ in $\Gamma(\pi)$ if, and only if there is a path from $(1, v)$ to $(n, w)$ in $\gamma(\pi)$, where $n=|\pi|$. Nodes are labelled by the regions they belong to. For any cycle $\pi$, we define $\Gamma(\pi)$ similarly on the node set $\mathcal{V}($ first $(\pi))$. Thus $\Gamma(\pi)$ may contain cycles; an example is given in Fig. 2 (on the right). We extend the operator $\oplus$ to folded orbit graphs. A strongly connected component (SCC) of a graph is initial if it is not reachable from any other SCC.

A forgetful cycle of $\mathcal{R}(\mathcal{A})$ is a cycle whose folded orbit graph is strongly connected. A cycle $\pi$ is aperiodic if for all $k \geq 1, \pi^{k}$ is forgetful. A lasso is said to be aperiodic if its cycle is. Note that there exist forgetful cycles that are not aperiodic [Sta12].

An example of a non-forgetful cycle is given in Fig. 3. The timed automaton of Fig. 1(b) contains a forgetful cycle, shown on Fig. 4.

Some Linear Algebra. For any set of vectors, we denote by $\operatorname{Span}(\mathcal{B})$ the linear span of $\mathcal{B}$, i.e. the set of linear combinations of $\mathcal{B}$. In the proofs, we will often use the vertices of a region to define a basis of a vector space that contains the region. The proof of the following lemma is given in Appendix B.

Lemma 2. Let $r$ be any region, and let $v_{0}=\inf (r)$. The set of vectors $\mathcal{B}_{v_{0}}=$ $\{v-\inf (r)\}_{v \in \mathcal{V}(r) \backslash\left\{v_{0}\right\}}$ is linearly independent. Moreover, the affine space $v_{0}+$ $\operatorname{Span}\left(\mathcal{B}_{v_{0}}\right)$ contains $r$.

Let the dimension of a subset $r \subseteq \mathbb{R}^{\mathcal{C}}$ be the least $d$ such that a affine subspace of $\mathbb{R}^{\mathcal{C}}$ of dimension $d$ contains $r$. It follows immediately from Lemma 2 that in any region where the partition of the clocks according to their fractional values is written as $X_{0}, X_{1}, \ldots, X_{m}$, has dimension $m$ since it has $m+1$ vertices.

We will consider the usual $d_{\infty}$ metric on $\mathbb{R}^{\mathcal{C}}$, defined as $d_{\infty}\left(\nu, \nu^{\prime}\right)=\max _{x \in \mathcal{C}} \mid \nu(x)$ $\nu^{\prime}(x) \mid$. We denote open balls in this metric by $\operatorname{Ball}_{d_{\infty}}(\nu, \varepsilon)$.

\section{Main Lemma and Algorithm}

Our main result is based on the following lemma, which gives a characterization of robust timed automata using aperiodic lassos of region automata.

Lemma 3 (Main Lemma). For any timed automaton $\mathcal{A}$ and Büchi objective $B$, there exists $\delta>0$ such that Controller has a winning strategy in $\mathcal{G}_{\delta}(\mathcal{A})$ for objective $B$, if, and only if $\mathcal{R}(\mathcal{A})$ has a reachable aperiodic non-punctual $B$ winning lasso. 
The algorithm for deciding robust Büchi acceptance follows from Lemma 3. It consists in looking for aperiodic non-punctual $B$-winning lassos in $\mathcal{R}(\mathcal{A})$. These lassos need not be simple, but the following lemma bounds their lengths.

Lemma 4. Let $B$ be a Büchi objective in a timed automaton $\mathcal{A}$, and $\pi$ be an aperiodic non-punctual B-winning cycle of $\mathcal{R}(\mathcal{A})$. Then, there exists a cycle $\pi^{\prime}$ with the same properties, with length at most $N=2^{(|\mathcal{C}|+1)^{2}+1} \times|\mathcal{R}(\mathcal{A})|$.

The polynomial-space algorithm then consists in guessing an accepting lasso of exponential size in $\mathcal{R}(\mathcal{A})$ on-the-fly, and checking whether its folded orbit graph is aperiodic. The folded orbit graph can also be computed on-the-fly, and aperiodicity can be checked in PSPACE [Sta12]. See Appendix for more details.

The two directions of the main lemma are proved using different techniques; they are presented respectively in Sections 5 and 6. Our results also establish that winning strategies can be represented by regions with a given granularity, depending on $\delta$. An algorithm is described at the end of Section 6 to actually compute the bound $\delta$, and a description of the winning strategy for Controller.

\section{$5 \quad$ No Aperiodic Lassos Implies No Robustness}

In this section, we prove that Controller loses if there is no aperiodic winning lassos. The idea is that if no accepting lasso of $\mathcal{R}(\mathcal{A})$ is aperiodic, then, as we show, the projection of any play to $\mathcal{R}(\mathcal{A})$ eventually enters and stays in a non-forgetful cycle. Then, we choose an appropriate Lyapunov function $L_{I}(\cdot)$ defined on valuations and taking nonnegative values, and describe a strategy for Perturbator such that the value of $L_{I}(\cdot)$ is decreased by at least $\varepsilon$ at each iteration of the cycle. Hence, Controller cannot cycle infinitely on such cycles: either it reaches a deadlock, or it cycles on non-accepting lassos. In the rest of this section, we describe Perturbator's strategy, study its outcomes, choose a function $L_{I}(\cdot)$, and prove the first direction of the main lemma.

Our proof is based on several results. First, we consider a result from Puri [Pur00](Lemma 5) on reachability relations between valuations in timed automata, and establish non-trivial properties on it valid along non-punctual paths. We then study the folded orbit graphs of non-punctual progress cycles, and use original proof techniques (e.g. using the dimension of sets) to understand the form of these graphs. This allows us to consider the Lyapunov functions of [BA11] in this context, and prove our results as described above.

\subsection{Reachability Relations}

We already noted that any valuation $\nu$ can be written as the convex combination of the vertices of its region, i.e. $\nu=\sum_{v \in \mathcal{V}([\nu])} \lambda_{v} v$ for some unique coefficients $\lambda_{v} \geq 0$ with $\sum_{v} \lambda_{v}=1$. When the region is clear from context, we will simply write $\nu=\lambda \boldsymbol{v}$. Given a path $\pi$ and a vertex $v$ of the region of first $(\pi)$, let us denote by $R_{\Gamma(\pi)}(v)$ the set of nodes $w \in \mathcal{V}(\operatorname{last}(\pi))$ such that $(v, w) \in E(\Gamma(\pi))$. Thus, this is the "image" of $v$ by the path $\pi$. Puri showed in [Pur00] that the reachability along paths can be characterized using orbit graphs. 
Lemma 5 ([Pur00]). Let $\pi$ be a path from region $r$ to $s$. Consider any $\nu \in r$ with $\nu=\sum_{v \in \mathcal{V}(r)} \lambda_{v} v$ for some coefficients $\lambda_{v} \geq 0$ and $\sum \lambda_{v}=1$. If $\nu \stackrel{\pi}{\rightarrow} \nu^{\prime}$, then for each $v \in \mathcal{V}(r)$, there exists a probability distribution $\left\{p_{v, w}^{\nu, \nu^{\prime}}\right\}_{w \in R_{\Gamma(\pi)}(v)}$ over $R_{\Gamma(\pi)}(v)$ such that $\nu^{\prime}=\sum_{v \in \mathcal{V}(r)} \lambda_{v} \sum_{w \in R_{\Gamma(\pi)}(v)} p_{v, w}^{\nu, \nu^{\prime}} w$. Conversely, if there exist probability distributions $p_{v, w}^{\nu, \nu^{\prime}}$ satisfying above equalities, then $\nu \stackrel{\bar{\pi}}{\rightarrow} \nu^{\prime}$.

Intuitively, the lemma shows that any successor of a point $\nu=\sum_{i} \lambda_{i} v_{i}$ can be obtained by distributing the weight $\lambda_{i}$ of each vertex $v_{i}$ to its successors following a probability distribution.

Example 1. The automaton of Fig. 1(a) contains a cycle on the region $r=\llbracket 1<$ $x, y<2 \wedge 0<x-y<1 \rrbracket$. The vertices of $r$ are $v_{1}=(1,1), v_{2}=(2,1), v_{3}=$ $(2,2)$. Consider a point $\nu=\frac{1}{3} v_{1}+\frac{1}{3} v_{2}+\frac{1}{3} v_{3}$. Then, Lemma 5 says that $\nu^{\prime}=$ $\sum_{1 \leq i \leq 3} \lambda_{i} v_{i}$ is reachable from $\nu$ along the cycle, where $\lambda_{1}=\frac{1}{3} 0.5+\frac{1}{3} 0.4=\frac{9}{30}$, $\lambda_{2}=\frac{1}{3} 1+\frac{1}{3} 0.3=\frac{13}{30}$, and $\lambda_{3}=\frac{1}{3} 0.6+\frac{1}{3} 0.2=\frac{4}{15}$. Here, vertex set $\left\{v_{1}, v_{3}\right\}$ is an initial SCC $I$. One can check that $L_{I}$ is indeed decreasing: $\frac{1}{3}+\frac{1}{3} \geq \frac{9}{30}+\frac{4}{15}$.

For any region $r$, and any subset $I \subseteq \mathcal{V}(r)$, we define the function $L_{I}: \bar{r} \rightarrow \mathbb{R}_{\geq 0}$ as $L_{I}(\nu)=\sum_{v \in I} \lambda_{v}$, where $\nu=\boldsymbol{\lambda} \boldsymbol{v}$. It is shown in [BA11] that given any cycle $\pi$, if $I$ is chosen as the initial strongly connected component of $\Gamma(\pi)$, then for any run $\nu \stackrel{\pi}{\rightarrow} \nu^{\prime}, L_{I}\left(\nu^{\prime}\right) \leq L_{I}(\nu)$. We will abusively use $L_{I}(\cdot)$ for a subset $I$ of nodes of $\gamma(\pi)$ or $\Gamma(\pi)$, that correspond to a same region. Notice that $0 \leq L_{I}(\cdot) \leq 1$.

\subsection{A global strategy for Perturbator}

Let us call a valuation $v \varepsilon$-far if $v+[-\varepsilon, \varepsilon] \subseteq[v]$. A run is $\varepsilon$-far if all delays end in $\varepsilon$-far valuations. We show that Perturbator has a strategy ensuring $\varepsilon$-far runs.

Lemma 6. Given any $\delta>0$, and any timed automaton with $C$ clocks, there exists a strategy $\sigma_{\delta,+}^{P}\left(\right.$ resp. $\left.\sigma_{\delta,-}^{P}\right)$ for Perturbator that always perturbs by a positive (resp. negative) amount, and whose all outcomes are $\frac{\delta}{2(C+1)}-$ far, and all delays are at least $\frac{\delta}{2(C+1)}$.

Proof. After any delay $\nu \stackrel{d}{\rightarrow} \nu^{\prime}, d \geq \delta$, chosen by Controller, consider the regions spanned by the set $\nu^{\prime}+[0, \delta]$. It is easy to see that this set intersects at most $|\mathcal{C}|+1$ different regions (See also [BMS12, Lemma 6]), all of which must satisfy the guard by definition of the game. So some region $r$ satisfies $\nu^{\prime}+[\alpha, \beta] \subseteq r$, for some $0 \leq \alpha<\beta \leq \delta$ with $\beta-\alpha \geq \frac{\delta}{|\mathcal{C}|+1}$. The strategy $\sigma_{\delta,+}^{\mathrm{P}}$ consists in choosing the perturbation as $\frac{1}{2}(\beta-\alpha)$. This guarantees time progress (of at least $\frac{\delta}{2(|\mathcal{C}|+1)}$ ). Moreover, the resulting valuation is always $\varepsilon$-far in its region, where $\varepsilon=\frac{\delta}{2(|\mathcal{C}|+1)}$. Observe also that all perturbations prescribed by this strategy are positive. The strategy $\sigma_{\delta,-}^{\mathrm{P}}$ is constructed similarly by considering the valuations $\nu^{\prime}+[-\delta, 0]$.

It turns out that in order to win, Perturbator only needs to ensure $\varepsilon$-far runs, hence either of the strategies defined above is sufficient to win. In the rest, let us 
fix a strategy $\sigma_{\delta}^{\mathrm{P}}$ as $\sigma_{\delta,+}^{\mathrm{P}}$ or $\sigma_{\delta,-}^{\mathrm{P}}$. In order to prove that $\varepsilon$-far runs are winning for Perturbator, we study the properties of the runs Outcome ${ }_{\mathcal{A}}^{\delta}\left(\cdot, \sigma_{\delta}^{\mathrm{P}}\right)$. We prove Propositions 1 and 2 which are a key element of the proof.

Using the $\varepsilon$-far property of the runs, the following proposition derives a bound on the convex combination coefficients of all visited valuations.

Proposition 1. Let $\rho \in$ Outcome $_{\mathcal{A}}\left(\cdot, \sigma_{\delta}^{P}\right)$. For any $i \geq 1$, if we write state $_{i}(\rho)=$ $\boldsymbol{\lambda} \boldsymbol{v}$, then $\lambda_{v} \geq \varepsilon$ for all vertices $v \in \mathcal{V}\left(\left[\right.\right.$ state $\left.\left._{i}(\rho)\right]\right)$.

Intuitively, a lower bound on the convex coefficients means that the valuation is not close to the borders of the region. We sketch the proof which is by induction. The property is true initially since the region $\mathbf{0}$ has a single vertex. For the induction case, let us mention the easy case of resets. Clock resets map each vertex to a single vertex, so each vertex has a single successor in the orbit graph. Then, it follows from Lemma 5 that the coefficient of each vertex in

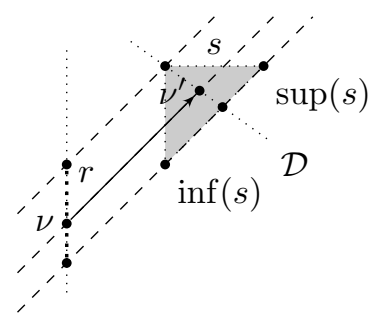
the target region is at least as large as its predecessor in the source region. For the case of time delays, one needs to consider the geometry of regions. The figure on the right shows the intuition in two dimensions. Given an $\varepsilon$-far delay from $\nu$ to $\nu^{\prime}$, with $[\nu]=r$, and $\left[\nu^{\prime}\right]=s$, one shows that the coefficients of $\inf (s)$ and $\sup (s)$ cannot be too small; otherwise the line $\mathcal{D}$ that connects $\nu^{\prime}$ to the third vertex would be close to vertical or to horizontal, requiring $\nu^{\prime}$ to be close to a border of $s$.

We need another property of similar spirit stating that all edges of the folded orbit graph receive a probability of at least $\min \left(\frac{1}{2}, \frac{\varepsilon}{2}\right)$ along $\varepsilon$-far delays, according to the interpretation of Lemma 5 . The proof is rather involved, and establishes that there is some degree of freedom in the choice of the probabilities of Lemma 5 .

Proposition 2. Let $\nu=\boldsymbol{\lambda} \boldsymbol{v}$ and $\nu^{\prime}=\boldsymbol{\lambda}^{\prime} \boldsymbol{v}^{\prime}$ denote two valuations satisfying $\boldsymbol{\lambda}, \boldsymbol{\lambda}^{\prime} \geq \varepsilon$, and s.t. $(\ell, \nu) \stackrel{\pi}{\rightarrow}\left(\ell, \nu^{\prime}\right)$ is an $\varepsilon$-far delay of duration at least $\varepsilon$. Then, for each $v \in \mathcal{V}([\nu])$, there exists a probability distribution $\left\{p_{v, w}\right\}_{w \in R_{\Gamma(\pi)}(v)}$ over $R_{\Gamma(\pi)}(v)$ s.t. $\nu^{\prime}=\sum_{v \in \mathcal{V}(r)} \lambda_{v} \sum_{w \in R_{\Gamma(\pi)}(v)} p_{v, w} w$, and $p_{v, w} \geq \min \left(\frac{1}{2}, \frac{\varepsilon}{2}\right)$.

\subsection{Decreasing Lyapunov function}

In the previous subsection, we established lower bounds on the convex coefficients of the visited valuations, and the probabilities of Lemma 5. We use this property to find a Lyapunov function that strictly decreases at each iteration of a cycle.

In this subsection, we concentrate on progress cycles. In fact, in the proof of Lemma 3, we will show that if Controller is able to win against $\sigma_{\delta}^{\mathrm{P}}$, then some cycle of $\mathcal{R}(\mathcal{A})$ must be repeated infinitely often. But since $\sigma_{\delta}^{\mathrm{P}}$ always ensures a time progress of $\frac{\delta}{2(|\mathcal{C}|+1)}$ (see Lemma 6$)$, if some clock is never reset, then its value would eventually be larger than the maximal constant, which is a contradiction since all clocks are assumed to be bounded. 
The following lemma shows that runs along non-punctual progress cycles can be modified so as to reach any valuation in a ball around the target state. This gives the dimension of the set of valuations reachable along a progress cycle starting from a given valuation. The result and its proof are similar in spirit to [DDMR08, Lemma 29].

Lemma 7. Let $\pi$ be a non-punctual progress cycle, and $(\ell, \nu) \stackrel{\pi}{\rightarrow}\left(\ell, \nu^{\prime}\right)$ a run along $\pi$. Then, there exists $\varepsilon>0$ such that there exists a run from $(\ell, \nu)$, along $\pi$, to any point in $\{\ell\} \times\left(\right.$ Ball $\left._{d_{\infty}}\left(\nu^{\prime}, \varepsilon\right) \cap\left[\nu^{\prime}\right]\right)$.

We now prove that the folded orbit graphs of non-punctual progress cycles are always connected. If the cycle is non-forgetful, there are at least two connected SCCs (Corollary 1). The lemma is proved by contradiction: we show that if the graph has disjoint components, then the set of states reachable from a given state cannot have full dimension, which contradicts Lemma 7.

Lemma 8. The folded orbit graph of any non-punctual progress cycle is connected.

Corollary 1. The folded orbit graph of a non-punctual non-forgetful progress cycle $\pi$ contains at least two strongly connected components that are connected. We associate with each $\pi$ an initial SCC of $\Gamma(\pi)$, which we denote by $I(\pi)$.

Hence, for any non-punctual non-forgetful cycle $\pi$, we consider the function $L_{I(\pi)}$. The following lemma shows a key property for the proof. It shows that $L_{I(\pi)}$ decreases by at least a fixed amount at each iteration of such a cycle under Perturbator's strategy $\sigma_{\delta}^{\mathrm{P}}$.

Lemma 9. Let $\omega \in$ Outcome $_{\mathcal{A}}^{\delta}\left(\cdot, \sigma_{\delta}^{P}\right)$, and $\rho$ be a finite prefix of $\omega$ such that $\pi$, the projection of $\rho$ to regions, is a cycle. If $\pi$ is a non-forgetful progress cycle, then, writing first $(\rho)=\boldsymbol{\lambda} \boldsymbol{v}$ and last $(\rho)=\boldsymbol{\lambda}^{\prime} \boldsymbol{v}^{\prime}$, we have, $\sum_{i \in I(\pi)} \lambda_{i}^{\prime} \leq \sum_{i \in I(\pi)} \lambda_{i}-\varepsilon^{2} / 2$.

The proof shows that any such run has a transition in which some edge of the folded orbit graph has a successor to a node that is not coreachable from $I(\pi)$. The existence of such an edge is proved using Corollary 1. Then, by Propositions 1 and 2, we know that the convex combination coefficient associated to the node is at least $\varepsilon$, and the probability associated to the edge leaving it is at least $\varepsilon / 2$. This allows showing that at least $\varepsilon^{2} / 2$ is lost from the sum $L_{\pi}$ at each iteration.

The previous lemma already gives an insight into the proof, since it follows that no non-forgetful cycle can be repeated infinitely under strategy $\sigma_{\delta}^{\mathrm{P}}$. However, one also needs to show that switching between different cycles cannot help Controller win. Thus, the last tool we need for the proof is the following factorization theorem, which allows factoring paths to cycles with the same folded orbit graphs.

Lemma 10. Let $\pi$ be a path of $\mathcal{R}(\mathcal{A})$ written as $\pi=\pi_{0} \pi_{1} \pi_{2} \ldots \pi_{n}$ where each $\pi_{i}$ is a cycle that starts in the same state, for $i \geq 1$. Then, one can write $\pi=\pi_{0}^{\prime} \pi_{1}^{\prime} \pi_{2}^{\prime} \ldots \pi_{m+1}^{\prime}$ such that $m \geq \sqrt{n / r-2}-1$, where $r=2^{(|\mathcal{C}|+1)^{2}}|\mathcal{R}(\mathcal{A})|$, and for some indices $0=\alpha_{0}<\alpha_{1}<\ldots$, we have $\pi_{i}^{\prime}=\pi_{\alpha_{i}} \cdot \ldots \cdot \pi_{\alpha_{i+1}-1}$ for each $i \geq 0$, and $\Gamma\left(\pi_{1}^{\prime}\right)=\Gamma\left(\pi_{i}^{\prime}\right)$ for all $1 \leq i \leq m$. 
Proof: No winning aperiodic lassos implies no robust safety. To get a contradiction, fix any winning strategy $\sigma$ for Controller and let $\rho$ be the infinite run Outcome ${ }_{\mathcal{A}}^{\delta}\left(\sigma, \sigma_{\delta}^{\mathrm{P}}\right)$, and $\pi$ its projection on regions. By definition of $\sigma_{\delta}^{\mathrm{P}}, \pi$ is a non-punctual path. Let us write $\pi=\pi_{0} \pi_{1} \ldots \pi_{n} \ldots$ such that all $\pi_{i}, i \geq 1$, are accepting cycles from a same state. Let $r=2^{(|\mathcal{C}|+1)^{2}} \times|\mathcal{R}(\mathcal{A})|, n=\left\lceil 2 / \varepsilon^{2}\right\rceil+1$ and $N$ large enough so that $\lceil\sqrt{N / r-2}\rceil-1 \geq n 2^{(|\mathcal{C}|+1)^{2}}$. We extract $\pi^{\prime}$ the prefix of $\pi$ with $N$ factors, and apply Lemma 10 which yields $\pi^{\prime}=\pi_{0}^{\prime} \pi_{1}^{\prime} \ldots \pi_{n^{\prime}}^{\prime} \pi_{n^{\prime}+1}^{\prime}$, with $n^{\prime}=n 2^{(|\mathcal{C}|+1)^{2}}$, where $\Gamma\left(\pi_{1}^{\prime}\right)=\ldots=\Gamma\left(\pi_{n^{\prime}}^{\prime}\right)$, and $\pi_{i}^{\prime}$ are obtained by concatenating one or several consecutive $\pi_{i}$. By hypothesis, some power $k$ of $\pi_{i}^{\prime}$ is non-forgetful, with $k \leq 2^{(|\mathcal{C}|+1)^{2}}$ since this is the number of folded orbit graphs for a fixed labelling function. But since the folded orbit graphs are the same for all $\pi_{i}^{\prime}$, this power is the same for all factors, and $\Gamma\left(\pi_{i}^{\prime k}\right)=\Gamma\left(\pi_{i}^{\prime} \pi_{i+1}^{\prime} \ldots \pi_{i+k-1}^{\prime}\right)$. Hence, we can factorize $\pi^{\prime}$ again into $\pi^{\prime}=\pi_{0}^{\prime} \pi_{1}^{\prime \prime} \pi_{2}^{\prime \prime} \ldots \pi_{n}^{\prime \prime} \pi_{n+1}^{\prime \prime}$, where $\Gamma\left(\pi_{1}^{\prime \prime}\right)=\ldots=$ $\Gamma\left(\pi_{n}^{\prime \prime}\right)$ and all are non-forgetful; while $\pi_{0}^{\prime}$ and $\pi_{n+1}^{\prime \prime}$ are arbitrary non-punctual paths. Moreover, $\pi_{i}^{\prime \prime}$, for $1 \leq i \leq n$, must be progress cycles too. In fact, otherwise there is some clock $x \in \mathcal{C}$ that is never reset along $\pi^{\prime}$. But because $\sigma_{\delta}^{\mathrm{P}}$ ensures a time progress of $\varepsilon$ at each delay, this means that $\pi^{\prime}$ contains delays of duration at least $n \varepsilon^{2} / 2>1$, so we cannot have first $\left(\pi_{1}^{\prime}\right)=\operatorname{last}\left(\pi_{n}^{\prime}\right)$ since the integer part of the clock $x$ changes, and so does the region since all clocks are assumed to be bounded. Now, we have $I\left(\pi_{i}^{\prime \prime}\right)=I\left(\pi_{j}^{\prime \prime}\right)$ for any $1 \leq i, j \leq n$, so the functions $L_{I\left(\pi_{i}^{\prime \prime}\right)}$ are the same for all $1 \leq i \leq n$. If we write $\rho_{i}$ the state reached in $\rho$ following $\pi_{0}^{\prime} \pi_{1}^{\prime \prime} \ldots \pi_{i}^{\prime \prime}$, then we get, by Lemma $9, L_{I\left(\pi_{1}^{\prime \prime}\right)}\left(\rho_{n}\right) \leq L_{I\left(\pi_{1}^{\prime \prime}\right)}\left(\rho_{0}\right)-n \varepsilon^{2} / 2$. This is a contradiction since $0 \leq L_{I\left(\pi_{1}^{\prime}\right)} \leq 1$ and $n \varepsilon^{2} / 2>1$.

\section{Aperiodic Lassos Implies Robustness}

We now prove that if $\mathcal{R}(\mathcal{A})$ contains an aperiodic non-punctual $B$-winning lasso, then there exists $\delta>0$ and a strategy for Controller in $\mathcal{G}_{\delta}(\mathcal{A})$ ensuring robust safety. The idea of the proof is to observe that aperiodic cycles do not constrain runs in the way non-forgetful ones do, and show that this is still the case in the perturbation game semantics. More precisely, along an aperiodic lasso, Controller is able to always come back in a set at the middle of the starting region; thus it can prevent perturbations from accumulating. As tools, we will use shrinking techniques from [SBM11,BMS12], the metric of [GHJ97] on timed traces.

\subsection{Zones and Shrunk Zones}

A zone is a subset of $\mathbb{R}_{>0}^{\mathcal{C}}$ defined by a guard. A difference-bound matrix (DBM) is a $\left|\mathcal{C}_{0}\right| \times\left|\mathcal{C}_{0}\right|$-matrix over $(\mathbb{R} \times\{<, \leq\}) \cup\{(\infty,<)\}$. We adopt the following notation: for any DBM $M$, we write $M=\left(\mathrm{M}, \prec^{M}\right)$, where $\mathrm{M}$ is the matrix made of the first components, with elements in $\mathbb{R} \cup\{\infty\}$, while $\prec^{M}$ is the matrix of the second components, with elements in $\{<, \leq\}$. A DBM $M$ naturally represents a zone (which we abusively write $M$ as well), defined as the set of valuations $v$ such that, for all $x, y \in \mathcal{C}_{0}$, it holds $v(x)-v(y) \prec_{x, y}^{M} \mathrm{M}_{x, y}$ (where $v(0)=0$ ). Standard operations used to explore the state space of timed automata have been defined on DBMs: intersection is written $M \cap N$, Pre $(M)$ is the set of time predecessors 
of $M$, Unreset $_{R}(M)$ is the set of valuations that end in $M$ when the clocks in $R$ are reset. We also consider $\operatorname{Pre}_{>\delta}(M)$, the set of time predecessors with a delay of more than $\delta$. DBMs were introduced in [BM83,Dil90] for analyzing timed automata; we refer to [BY04] for details.

A parametrised extension, namely shrunk DBMs were introduced in [SBM11] in order to study the parametrised state space of timed automata. Intuitively, our goal is to express shrinkings of guards, e.g. sets of states satisfying constraints of the form $g=1+\delta<x<2-\delta \wedge 2 \delta<y$, where $\delta$ is a parameter to be chosen. Formally, a shrunk DBM is a pair $(M, P)$, where $M$ is a DBM, and $P$ is a nonnegative integer matrix called a shrinking matrix (SM). This pair represents the set of valuations defined by the DBM $M-\delta P$, for any given $\delta>0$. Considering the example $g, M$ is the guard $g$ obtained by setting $\delta=0$, and $P$ is made of the integer multipliers of $\delta$.

We adopt the following notation: when we write a statement involving a shrunk DBM $(M, P)$, we mean that for some $\delta_{0}>0$, the statement holds for $(M-\delta P)$ for all $\delta \in\left[0, \delta_{0}\right]$. For instance, $(M, P)=\operatorname{Pre}_{>\delta}((N, Q))$ means that $M-\delta P=\operatorname{Pre}_{>\delta}(N-\delta Q)$ for all small enough $\delta>0$. Additional operations are defined for shrunk DBMs: for any $(M, P)$, we define shrink ${ }_{[-\delta, \delta]}((M, P))$ as the set of valuations $\nu$ such that $\nu+[-\delta, \delta] \subseteq M-\delta P$, for small enough $\delta>0$.

Shrunk DBMs are closed under standard operations on zones:

Lemma 11 ([SBM11,BMS12]). Let $M=f\left(N_{1}, \ldots, N_{k}\right)$ be an equation between normalized DBMs $M, N_{1}, \ldots, N_{k}$, using the operators Pre $>\delta$, Unreset ${ }_{R}, \cap$, and shrink ${ }_{[-\delta, \delta]}$, and let $P_{1}, \ldots, P_{k}$ be $S M$ s. Then, there exists a shrunk DBM $\left(M^{\prime}, Q\right)$ with $\mathrm{M}^{\prime}=\mathrm{M}, M \subseteq M^{\prime}$ and $\left(M^{\prime}, Q\right)=f\left(\left(N_{1}, P_{1}\right), \ldots,\left(N_{k}, P_{k}\right)\right)$. The shrunk DBM $\left(M^{\prime}, Q\right)$ and an upper bound on $\delta$ can be computed in poly-time.

\subsection{Controllable Predecessors}

Consider an edge $e=\left(\ell, g, R, \ell^{\prime}\right)$. For any set $Z \subseteq \mathbb{R}_{\geq 0}^{\mathcal{C}}$, we define the controllable predecessors of $Z$ as follows: $\operatorname{CPre}_{e}^{\delta}(Z)=\operatorname{Pre}_{>\delta}\left(\operatorname{shrink}_{[-\delta, \delta]}\left(g \cap \operatorname{Unreset}_{R}(Z)\right)\right)$. Intuitively, $\operatorname{CPre}_{e}^{\delta}(Z)$ is the set of valuations from which Controller can ensure reaching $Z$ in one step, following the edge $e$. In fact, it can delay in shrink $_{[-\delta, \delta]}\left(g \cap \operatorname{Unreset}_{R}(Z)\right)$ with a delay of more than $\delta$, where under any perturbation in $[-\delta, \delta]$, the valuation satisfies the guard, and it ends, after reset, in $Z$. We extend this operator to paths as expected. Note that $\mathrm{CPre}_{e}^{0}$ is the usual predecessor operator without perturbation: If $N=\mathrm{CPre}_{\pi}^{0}(M)$ for some sets $N, M$ and path $\pi$, then, $N$ is the set of all valuations that can reach some valuation in $M$ following $\pi$.

It immediately follows from Lemma 11 that the controllable predecessors of shrunk DBMs are shrunk DBMs, which are computable.

Corollary 2. Let $e=\left(\ell, g, R, \ell^{\prime}\right)$ be an edge. Let $M$ and $N$ be non-empty DBMs such that $N=\mathrm{CPre}_{e}^{0}(M)$. Then, for any $S M P$, there exists an $S M Q$ such that $\left(N^{\prime}, Q\right)=\operatorname{CPre}_{e}^{\delta}((M, P))$ for some $N \subseteq N^{\prime}$ and $\mathrm{N}=\mathrm{N}^{\prime}$.

The set $\left(N^{\prime}, Q\right)$ given by the previous lemma can be empty in general. However, as the following lemma shows, it turns out that in the case of non-punctual paths, controllable predecessors of open sets are non-empty, for small enough $\delta>0$. 
Lemma 12. Let $\pi$ be a non-punctual path from region $r$ to $s$. Let $s^{\prime} \subseteq s$ such that there exists $\nu^{\prime} \in s^{\prime}$ and $\varepsilon>0$ with Ball $d_{\infty}\left(\nu^{\prime}, \varepsilon\right) \cap s \subseteq s^{\prime}$. Then, $\operatorname{CPre}_{\pi}^{\delta}\left(s^{\prime}\right)$ is non-empty for small enough $\delta>0$.

\subsection{Winning Under Perturbations}

Let $\pi_{0} \pi$ denote a non-punctual aperiodic lasso, where $\pi$ is the cycle; $\Gamma\left(\pi^{n}\right)$ is strongly connected for $n \geq 1$. We prove that the graph of some power is complete:

Lemma 13. Let $\pi$ be an aperiodic cycle. Then, there exists $n \leq\left|\mathcal{C}_{0}\right| \cdot\left|\mathcal{C}_{0}\right|$ ! such that $\Gamma\left(\pi^{n}\right)$ is a complete graph.

Let us assume, by the previous lemma, that $\Gamma(\pi)$ is a complete graph (one can consider the lasso $\pi_{0} \pi^{n}$ for an appropriate $n$ ). Let $s$ be a region with smaller granularity inside $r$, obtained so that the Hausdorff distance between $r$ and $s$ is positive. In this case, $s$ can be chosen so that it can be expressed by a DBM (with rational components). The construction is defined in the following lemma.

Lemma 14. For any non-empty DBM $M$, there exists a non-empty DBM $N$ such that Ball $_{d_{\infty}}(\nu, \varepsilon) \cap M \subseteq N$ for some $\nu \in M$, and for any shrinking matrix $P$ with $(M, P) \neq \emptyset, N \subseteq(M, P)$. Moreover, $N$ is computable in polynomial time.

The last element we need for our proof is an observation from [BA11]: If $\pi$ is a cycle of $\mathcal{R}(\mathcal{A})$ such that $\Gamma(\pi)$ is a complete graph, then for all $(\ell, \nu),\left(\ell, \nu^{\prime}\right) \in$ first $(\pi)$, there is a run $(\ell, \nu) \stackrel{\pi}{\rightarrow}\left(\ell, \nu^{\prime}\right)$, hence the reachability relation is complete.

Proof: Winning aperiodic lassos implies robust safety. We write $r=$ first $(\pi)$. Let $s \subseteq r$ given by Lemma 14 applied to $r$. Because $\Gamma(\pi)$ is complete, we have, by previous remark $r=\mathrm{CPre}_{\pi}^{0}(s)$. By Lemma 2, there exists a SM $Q$ such that $(r, Q)=\operatorname{CPre}_{\pi}^{\delta}(s)$. By Lemma $12,(r, Q)$ is non-empty. By definition of $s$, for small enough $\delta>0, s \subseteq(r, Q)$, so Controller has a strategy to always move inside $s$, at each iteration of $\pi$. Similarly, $\operatorname{CPre}_{\pi_{0}}^{\delta}((r, Q))$ is also non-empty and therefore contains the initial state. Hence, Controller wins.

Now, to actually compute $\delta$ and a winning strategy in exponential time, given an aperiodic lasso, one iterates the cycle so as to obtain a complete folded orbit graph (Lemma 13), then picks a subset $s$ as in Lemma 14, and uses Corollary 2 to compute the controllable predecessors of $s$. This also provides the greatest $\delta$ under which the strategy along given lasso is valid.

\section{Future works}

We intend to investigate possible extensions of this work to timed games, where Perturbator entirely determines the move in some locations. This could require generalizing the notion of aperiodicity from paths to trees since Controller can no more ensure to follow a given path. Another interesting future work is studying infinite runs in the semantics of [BMS12]. Although the proof techniques of [BMS12] are different, it could be possible to apply some of the ideas of this paper. 


\section{References}

[AD94] R. Alur and D. L. Dill. A theory of timed automata. Theoretical Computer Science, 126(2):183-235, 1994.

[BA11] N. Basset and E. Asarin. Thin and thick timed regular languages. In FORMATS'11, LNCS 6919, p. 113-128. Springer, 2011.

[BM83] B. Berthomieu and M. Menasche. An enumerative approach for analyzing time Petri nets. In WCC'83, p. 41-46. North-Holland/IFIP, 1983.

[BMS12] P. Bouyer, N. Markey, and O. Sankur. Robust reachability in timed automata: A game-based approach. In ICALP'12, LNCS 7392, p. 128-140, Warwick, UK, 2012. Springer.

[BY04] J. Bengtsson and W. Yi. Timed automata: Semantics, algorithms and tools. In Lectures on Concurrency and Petri Nets, LNCS 2098, p. 87-124. Springer, 2004.

[CHP11] K. Chatterjee, T. A. Henzinger, and V. S. Prabhu. Timed parity games: Complexity and robustness. Logical Methods in Computer Science, 7(4), 2011.

[CHR02] F. Cassez, T. A. Henzinger, and J.-F. Raskin. A comparison of control problems for timed and hybrid systems. In HSCC'02, LNCS 2289, p. 134-148. Springer, 2002.

[DDMR08] M. De Wulf, L. Doyen, N. Markey, and J.-F. Raskin. Robust safety of timed automata. Formal Methods in System Design, 33(1-3):45-84, 2008.

[DDR05] M. De Wulf, L. Doyen, and J.-F. Raskin. Almost ASAP semantics: From timed models to timed implementations. Formal Aspects of Computing, 17(3):319-341, 2005.

[Dil90] D. L. Dill. Timing assumptions and verification of finite-state concurrent systems. In AVMFSS'89, LNCS 407, p. 197-212. Springer, 1990.

[GHJ97] V. Gupta, Th. A. Henzinger, and R. Jagadeesan. Robust timed automata. In HART'97, LNCS 1201, p. 331-345. Springer, 1997.

[Pur00] A. Puri. Dynamical properties of timed automata. Discrete Event Dynamic Systems, 10(1-2):87-113, 2000.

[San11] O. Sankur. Untimed language preservation in timed systems. In MFCS'11, LNCS 6907, p. 556-567. Springer, 2011.

[SBM11] O. Sankur, P. Bouyer, and N. Markey. Shrinking timed automata. In FSTTCS'11, LIPIcs 13, p. 375-386. Leibniz-Zentrum für Informatik, 2011.

[Sta12] A. Stainer. Frequencies in forgetful timed automata. In FORMATS'12, LNCS 7595, p. 236-251. Springer, 2012. 


\section{A PSPACE-hardness of Parametrised Robust Control}

We show PSPACE-hardness of the parametrised robust controller synthesis problem, both for reachability and safety objectives.

We reduce the halting problem for linearly-bounded deterministic Turing machines to our synthesis problem. Let $\mathcal{M}$ be a linearly-bounded deterministic Turing machine, and $w_{0}$ be an input to $\mathcal{M}$. Let $N$ be the bound on the tape of $\mathcal{M}$ when simulating on input word $w_{0}\left(N\right.$ is linear in $\left.\left|w_{0}\right|\right)$. We assume the alphabet is $\{a, b\}$, and we encode the content of the $i$-th cell $C_{i}$ of $\mathcal{M}$ using a clock $x_{i}$ with the following convention: when the module is entered, cell $C_{i}$ contains an $a$ whenever $x_{i}<1$, and it contains a $b$ whenever $x_{i}>2$. To simulate a transition $\left(q, \alpha, q^{\prime}, \beta\right.$, dir) of $\mathcal{M}$, where $\alpha, \beta \in\{a, b\}$ and $\operatorname{dir} \in\{-1,1\}$, we build a module as in Fig. 5 for every index $i$ such that both $i$ and $i+\operatorname{dir}$ lie between 1 and $N$. Along this module, after any initial delay of duration $u_{0} \in(2,3)$, cell $C_{j}$ contains $a$ iff $x_{j}<4$, and it contains $b$ iff $x_{j}>4$. Transitions between $p_{j}$ and $p_{j+1}$, for $j \neq i$, ensure preservation of this encoding. Between $p_{i}$ and $p_{i+1}$, the module checks that $C_{i}=\alpha$ (through guard $g_{\alpha, i}$ ), and replace this content with $\beta$ (through reset $\left.Y_{\beta, i}\right)$. This way, one run through the module updates the content of the tape and the position of the tape head according to the selected transition of $\mathcal{M}$.

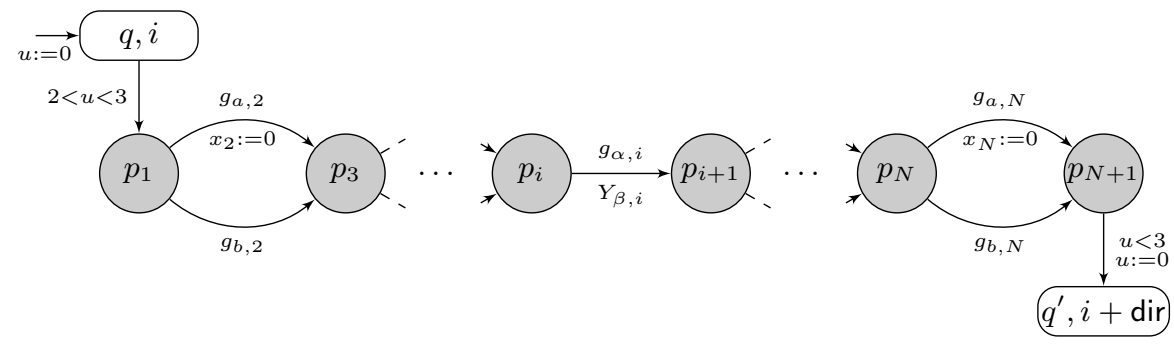

Fig. 5. Simulation transition $\left(q, \alpha, q^{\prime}, \beta\right.$, dir) where $\alpha, \beta \in\{a, b\}$. Index $i$ is such that $1 \leq i \leq N$ and $1 \leq i+\operatorname{dir} \leq N$. Guards and resets are defined as follows: $g_{a, j}$ is $\left(x_{j}<4 \wedge u<3\right)$ and $g_{b, j}$ is $\left(x_{j}>4 \wedge u<3\right)$, while $Y_{a, j}$ is $\left\{x_{j}\right\}$ and $Y_{b, j}$ is empty.

We complete the construction with an initialization module (encoding input $w_{0}$ on the tape). We write $\mathcal{A}$ for this timed automaton and write halt for the halting location (which is made a sink).

We show the following lemma:

Lemma 15. Let $s_{0}$ be the initial configuration of $\mathcal{A}$. The following three properties are equivalent:

1. $\mathcal{M}$ halts on $w_{0}$;

2. Controller has a robust strategy in $\mathcal{A}$ to reach halt, which can be perturbed by at most $\delta<\frac{1}{4 N}$

3. Controller has no robust strategy in $\mathcal{A}$ to avoid halt, whatever the value of $\delta>0$. 
Proof. We first prove the equivalence of the two first properties.

First assume that $\mathcal{M}$ halts on $w_{0}$. We describe a robust winning strategy for the Controller in $\mathcal{A}$. We consider the simulation of the transition $\left(q, \alpha, q^{\prime}, \beta\right.$, dir) of $\mathcal{M}$. Assume state $(q, i)$ is entered with some valuation $v_{0}$ that properly encodes some content of the tape of $\mathcal{M}$ : if cell $C_{j}$ contains an $a$, then $v_{0}\left(x_{j}\right)<1$, and if cell $C_{j}$ contains a $b$, then $v_{0}\left(x_{j}\right)>2$. We describe a strategy for Controller, and then pick a corresponding outcome (with perturbations). The candidate strategy is as follows: leave $(q, i)$ when $u=2.5$, and in each of the $p_{j}$ 's, wait for $\delta$ time units, and then takes the next feasible transition.

Under that strategy, and assuming that Perturbator perturbs by $-\delta \leq \varepsilon_{0} \leq \delta$ on transition leaving $(q, i)$, and by $-\delta \leq \varepsilon_{j} \leq \delta$ on transition leaving $p_{j}$, the valuation when arriving in $p_{j}$ is $v_{j}$ (resp. in $\left(q^{\prime}, i+\operatorname{dir}\right)$ is $\left.v_{0}^{\prime}\right)$ such that:

- if $i \neq j$ and cell $C_{j}$ contains a $b$, or if $i=j$ and $\beta=b$, then for every $1 \leq k \leq N+1, v_{k}\left(x_{j}\right)=v_{0}\left(x_{j}\right)+2.5+(k-1) \delta+\sum_{h=0}^{k-1} \varepsilon_{h} ;$

- if $i \neq j$ and cell $C_{j}$ contains an $a$, or if $i=j$ and $\beta=a$, then for every $1 \leq k \leq j, v_{k}\left(x_{j}\right)=v_{0}\left(x_{j}\right)+2.5+(k-1) \delta+\sum_{h=0}^{k-1} \varepsilon_{h}, v_{j+1}\left(x_{j}\right)=0$, and for every $j+2 \leq k \leq N+1, v_{k}\left(x_{j}\right)=(k-j-1) \delta+\sum_{h=j+1}^{k-1} \varepsilon_{h}$.

Note that in all cases, since $\delta<\frac{1}{4 N}$, if cell $C_{j}$ contains an $a$, then $v_{j}+\delta+\varepsilon_{j}=g_{a, j}$, and if cell $C_{j}$ contains a $b$, then $v_{j}+\delta+\varepsilon_{j} \models g_{b, j}$. Note also that if either $i \neq j$ and cell $C_{j}$ contains an $a$, or if $i=j$ and $\beta=a$, then $v_{0}^{\prime}\left(x_{j}\right)<1$, and if either $i \neq j$ and cell $C_{j}$ contains a $b$, or if $i=j$ and $\beta=b$, then $v_{0}^{\prime}\left(x_{j}\right)>2$. This means that whatever the perturbation, at the end of the module the clocks properly encode the new content of the tape of $\mathcal{M}$ after the mentioned transition has been taken.

We can apply this strategy in every module simulating a transition of the Turing machine. Since $\mathcal{M}$ halts on $w_{0}$, the halt state can be reached by following this strategy.

Conversely, if $\mathcal{M}$ does not halt on $w_{0}$, then when playing without perturbations, Controller cannot reach the halting state. In particular, Controller has no robust strategy.

Now we should realize that if the Turing machine halts on $w_{0}$, then every non-blocking (standard) execution in $\mathcal{A}$ leads to halt. So in particular, there is no robust strategy for avoiding halt. If the Turing machine does not halt, then the same strategy as above is robust and avoids halt. This implies the equivalence with the third property.

In the above construction some clocks may not be bounded. However we can modify the construction to ensure that all clocks are always bounded. We assume that an $a$ in cell $C_{j}$ is encoded with clock constraint $x_{j}<1$, and a $b$ with clock constraint $2<x_{j}<4$ when entering the module. Assuming this, the previous module ensures that, when leaving the module, either $x_{j}<1$ (in case cell $C_{j}$ now contains an $a$ ), or either $2<x_{j}<4$ or $4<x_{j}<7$ (the two possible cases for cell $C_{j}$ to contain a $b$ ). It is easy to plug at the end of the module another module, which ensures that $2<x_{j}<4$ when cell $C_{j}$ contains an $a$, and 
$x_{j}<1$ when cell $C_{j}$ contains a $b$. Then we can plug at the end of this second module another one which ensures that $x_{j}<1$ when cell $C_{j}$ contains an $a$, and $2<x_{j}<4$ when cell $C_{j}$ contains a $b$, which is the initial encoding. Using this trick, the constructed timed automata has only bounded clocks.

\section{B Proofs}

\section{B.1 Proofs of Section 3}

Lemma 2. Let $r$ be any region, and let $v_{0}=\inf (r)$. The set of vectors $\mathcal{B}_{v_{0}}=$ $\{v-\inf (r)\}_{v \in \mathcal{V}(r) \backslash\left\{v_{0}\right\}}$ is linearly independent. Moreover, the affine space $v_{0}+$ $\operatorname{Span}\left(\mathcal{B}_{v_{0}}\right)$ contains $r$.

Proof. Recall the definition of vertices of $r$ using the partition $X_{0}, X_{1}, \ldots, X_{m}$ of the clocks according to their fractional parts. The vertex $v_{0}=\inf (r)$ corresponds to the case where all the clocks are equal to their lower bounds. Region $r$ has one vertex $v_{i}$ for each $1 \leq i \leq m$, in which all clocks $X_{1}, \ldots, X_{i}$ are equal to their lower bounds, and the rest of them are equal to their upper bounds. Hence, one can write $v_{i}-v_{0}$ as a column vector of $\left|X_{0}\right|+\ldots+\left|X_{i}\right|$ zeros, followed by $\left|X_{i+1}\right|+\ldots+\left|X_{m}\right|$ ones, in the orthogonal basis of $\mathbb{R}^{\mathcal{C}}$. This set is clearly independent.

To see that the affine space $v_{0}+\operatorname{Span}\left(\mathcal{B}_{\inf (r)}\right)$ contains the entire region, write any $\nu \in r$ as the convex combination of the vertices of $r: \nu=\sum_{v \in \mathcal{V}(r)} \lambda_{v} v$. We get that $\nu-\inf (r)=\sum_{v \in \mathcal{V}(r)} \lambda_{v}(v-\inf (r))$, since $\sum_{v \in \mathcal{V}(r)} \lambda_{v} v-v_{0}=$ $\sum_{v \in \mathcal{V}(r)} \lambda_{v}\left(v-v_{0}\right)=\sum_{v \neq v_{0}} \lambda_{v}\left(v-v_{0}\right)$, using $\sum_{v} \lambda_{v}=1$.

Metric on Timed Traces. A timed trace is a sequence $\left(t_{i}, e_{i}\right)_{1 \leq i \leq n}$ where $t_{i} \geq 0$ and each $e_{i}$ is an edge. The timed trace of a run $\rho$ is the sequence $\operatorname{trace}(\rho)=$ $\left(t_{i}, e_{i}\right)_{1 \leq i \leq n}$, where the $t_{i} \geq 0$ are the delay transitions of $\rho$, and $e_{i}$ are the edges it takes. Given a path $\pi$ of $\mathcal{R}(\mathcal{A})$, and a state $q \in \operatorname{first}(\pi)$, we say that a timed trace $\left(t_{i}, e_{i}\right)_{1 \leq i \leq n}$ is feasible for $\pi$ and $q$ if there is a run starting at $q$, along $\pi$, whose timed trace is $\left(t_{i}, e_{i}\right)_{1 \leq i \leq n}$. We write untime $\left(\left(t_{i}, e_{i}\right)_{1 \leq i \leq n}\right)=\left(e_{i}\right)_{1 \leq i \leq n}$. For a path $\pi$ of $\mathcal{R}(\mathcal{A})$, and $q \in \operatorname{first}(\pi)$, let ttrace $(\pi, q)$ denote the set of timed traces that are feasible for $\pi$ and $q$.

We define a variant of the metric of [GHJ97] on timed traces, which defines the same topology. Given two timed traces $u=\left(t_{i}, e_{i}\right)_{1 \leq i \leq n}$ and $u^{\prime}=\left(t_{i}^{\prime}, e_{i}^{\prime}\right)_{1 \leq i \leq n}$, we let $d\left(u, u^{\prime}\right)=\infty$ if untime $(u) \neq \operatorname{untime}\left(u^{\prime}\right)$, and $\bar{d}\left(u, u^{\prime}\right)=\max \left\{\left|t_{i}-t_{i}^{\prime}\right|, 1 \leq\right.$ $i \leq n\}$ otherwise. We define $\operatorname{Ball}_{d}(u, \varepsilon)$ as the open ball of radius $\varepsilon$ around $u$ in this metric. Note that the original metric was defined using timestamps rather than delays, but both metrics define the same topology [GHJ97].

Proposition 3 ([GHJ97]). If $\pi$ is a non-punctual path, and $q \in$ first $(\pi)$, then $\operatorname{ttrace}(\pi, q)$ is an open set (for the topology induced by $d$ ).

\section{B.2 Proofs of Section 4}

Lemma 4. Let $B$ be a Büchi objective in a timed automaton $\mathcal{A}$, and $\pi$ be an aperiodic non-punctual B-winning cycle of $\mathcal{R}(\mathcal{A})$. Then, there exists a cycle $\pi^{\prime}$ with the same properties, with length at most $N=2^{(|\mathcal{C}|+1)^{2}+1} \times|\mathcal{R}(\mathcal{A})|$. 

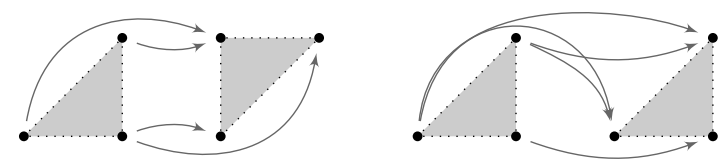

Fig. 6. Illustration of both cases of Lemma 17

Proof. We label all prefixes $\pi_{1 \ldots i}$ with $\left(\Gamma\left(\pi_{1 \ldots i}\right), b_{i}\right)$, where $b_{i}=\top$ if $\pi_{1 \ldots i}$ visits a location in $B$, and $\perp$ otherwise. The number of labels is at most $N=2^{(|\mathcal{C}|+1)^{2}+1} \times$ $|\mathcal{R}(\mathcal{A})|$, which is the number of folded orbit graphs. We argue that a cycle $\pi^{\prime}$ of length less than $N$ can be extracted from $\pi$, if $|\pi| \geq N$. In fact, in this case, we must have $i<j$ such that $\Gamma\left(\pi_{1 \ldots i}\right)=\Gamma\left(\pi_{1 \ldots j}\right)$ and $b_{i}=b_{j}$. But then, removing the infix $\pi_{i+1 \ldots j}$ yields a shorter cycle, which visits $B$ if $\pi_{1 \ldots j}$ does so, and with the same folded orbit graph. By repeating this procedure, we obtain a path $\pi^{\prime}$ of length less than $N$, which is non-punctual aperiodic $B$-winning.

The polynomial-space algorithm starts by guessing, using polynomial space, a state $q_{1}$ reachable by a non-punctual path in $\mathcal{R}(\mathcal{A})$. From $q_{1}$, one can guess an aperiodic non-punctual $B$-winning cycle in polynomial space as follows. We visit a path $q_{1} e_{1} q_{2} e_{2} \ldots q_{i}$ nondeterministically, and only keeping in memory the graph $G_{i}=\Gamma\left(q_{1} e_{1} q_{2} e_{2} \ldots q_{i}\right)$ and a boolean indicating whether a winning state has been visited. Thanks to the operator $\oplus$, for any transition $q_{i} e_{i} q_{i+1}$, one can compute $G_{i+1}$ using only $G_{i}$ and this transition. Whenever the state $q_{1}$ is visited again, the algorithm checks whether the current graph $G_{i}$ is aperiodic; this can be done in polynomial space as shown in [Sta12]. If so, the algorithm stops and accepts. Otherwise, the input is rejected after $N$ steps.

\section{B.3 Proofs of Section 5}

A global strategy for Perturbator We show Proposition 1 first for discrete transitions, then for $\varepsilon$-far delays. The following lemma treats the clock resets, thus discrete transitions. The proof follows from Lemma 5 using the fact that each node has a unique successor in the orbit graph of a reset. Here, $\min (\boldsymbol{\lambda})$ is the minimum among the components of $\boldsymbol{\lambda}$.

Lemma 16. For any $\nu=\boldsymbol{\lambda} \boldsymbol{v}$ and $\nu^{\prime}=\boldsymbol{\lambda}^{\prime} \boldsymbol{v}^{\prime}$ with $\nu^{\prime}=\nu[R \leftarrow 0]$ for some $R \subseteq \mathcal{C}$, $\min \left(\boldsymbol{\lambda}^{\prime}\right) \geq \min (\boldsymbol{\lambda})$.

Proof. The orbit graph of a clock reset has the property that all vertices of $[\nu]$ has outdegree 1 (since the reset defines a function). By Lemma 5, each $\lambda_{v_{i}^{\prime}}$ is equal to the sum of a subset of the components of $\boldsymbol{v}$, namely those vertices that have an edge to vertex $v_{i}^{\prime}$. The inequality follows. See Fig. 7 for an illustration.

Proving Proposition 1 for delays is slightly more difficult. The proof relies on the properties of the orbit graphs of non-punctual delays, as shown in the following lemma. 


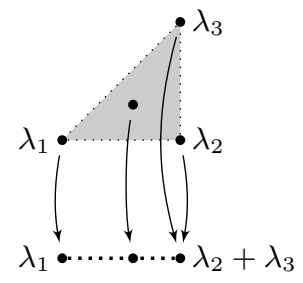

Fig. 7. Illustration of Lemma 16.

Lemma 17. If $\pi=r_{1} \Delta r_{2}$ is a non-punctual delay, then exactly one of the following cases must hold for the graph $\gamma(\pi)$ :

1. Exactly one vertex of $r_{1}$ has outdegree 2. The successors of this vertex are $\inf \left(r_{2}\right)$ and $\sup \left(r_{2}\right)$. All other vertices of $r_{1}$ have outdegree 1.

2. Exactly two vertices of $r_{1}$ have outdegree 2 . These vertices are $\inf \left(r_{1}\right)$ and $\sup \left(r_{1}\right)$ and both lead to $\inf \left(r_{2}\right)$ and $\sup \left(r_{2}\right)$. All other vertices of $r_{1}$ have outdegree 1 .

Moreover all vertices of $r_{2}$ have indegree at most 2, and the predecessors of any vertex of indegree 2 are $\inf \left(r_{1}\right)$ and $\sup \left(r_{1}\right)$.

We note the following easy lemma on $\varepsilon$-far valuations.

Lemma 18. If $\nu$ is an $\varepsilon$-far valuation, then for all clock $x$, if $l_{x}$ and $u_{x}$ denote respectively the lower and upper bounds on $x$ in region $[\nu]$, then $l_{x}+\varepsilon \leq \nu(x) \leq$ $u_{x}-\varepsilon$. It follows that for all $v \in \mathcal{V}([\nu]), d_{\infty}(v, \nu) \geq \varepsilon$.

Lemma 19. Let $\nu=\boldsymbol{\lambda} \boldsymbol{v}$ and $\nu^{\prime}=\boldsymbol{\lambda}^{\prime} \boldsymbol{v}^{\prime}$ where $\nu^{\prime}=\nu+d$ for some $d \geq 0$ and $\nu^{\prime}$ is $\varepsilon$-far. If $[\nu]$ is a punctual region, and $\min (\boldsymbol{\lambda}) \geq \varepsilon$, then $\min \left(\boldsymbol{\lambda}^{\prime}\right) \geq \varepsilon$.

Proof. We denote $r=[\nu]$ and $s=\left[\nu^{\prime}\right]$. Let $\gamma$ denote the orbit graph of the delay $r \rightarrow s$. Because we have an $\varepsilon$-far delay, $s$ must have vertices $\inf (s) \neq \sup (s)$.

We first show that $\lambda_{\inf (s)}^{\prime}, \lambda_{\sup (s)}^{\prime} \geq \varepsilon$. There exists a clock $x$ such that $\sup (s)(x)=v(x)+1$ for any other vertex $v$ of region $s$ ( $x$ is one of the first clocks that appear in the fractional ordering of the clocks inside $s)$. Let us denote by $l=\inf (s)(x)$ and $u=\sup (s)(x)$. By Lemma 18, we must have

$$
l+\varepsilon \leq \lambda_{\sup (s)}^{\prime} \cdot u+\sum_{v \neq \sup (s)} \lambda_{v}^{\prime} \cdot v(x) .
$$

Using the fact that $u=l+1$, and $v(x)=l$ for all $v \neq \sup (s)$, we get

$$
\begin{aligned}
l+\varepsilon & \leq \lambda_{\sup (s)}^{\prime} \cdot u+\sum_{v \neq \sup (s)} \lambda_{v}^{\prime} \cdot l, \\
l+\varepsilon & \leq \lambda_{\sup (s)}^{\prime}+l \sum_{v} \lambda_{v}^{\prime}, \\
\varepsilon & \leq \lambda_{\sup (s)}^{\prime} .
\end{aligned}
$$

A symmetric argument can be used to prove $\lambda_{\inf (s)}^{\prime} \geq \varepsilon$.

Consider now a vertex $v \notin\{\sup (s), \inf (s)\}$. Because $r$ is punctual, $v$ has exactly one predecessor $u$ in $r$. And since $v \neq \inf (s), \sup (s), u$ has no other successors than $v$ (see Lemma 17). Consequently, $\lambda_{v}^{\prime}=\lambda_{u}^{\prime}$. 
Proof (Proof of Proposition 1). By induction on the length of a run under strategy $\sigma_{\delta}^{\mathrm{P}}$. Initially, we are in a singleton region with one vertex, so the only convex coefficient is 1 . In the induction step, resets follow from Lemma 16. Delays follow from Lemma 19 since all delays are $\varepsilon$-far, and by the fact that the valuation after the latest reset lies in a punctual region.

Proposition 2. Let $\nu=\boldsymbol{\lambda} \boldsymbol{v}$ and $\nu^{\prime}=\boldsymbol{\lambda}^{\prime} \boldsymbol{v}^{\prime}$ denote two valuations satisfying $\boldsymbol{\lambda}, \boldsymbol{\lambda}^{\prime} \geq \varepsilon$, and s.t. $(\ell, \nu) \stackrel{\pi}{\rightarrow}\left(\ell, \nu^{\prime}\right)$ is an $\varepsilon$-far delay of duration at least $\varepsilon$. Then, for each $v \in \mathcal{V}([\nu])$, there exists a probability distribution $\left\{p_{v, w}\right\}_{w \in R_{\Gamma(\pi)}(v)}$ over $R_{\Gamma(\pi)}(v)$ s.t. $\nu^{\prime}=\sum_{v \in \mathcal{V}(r)} \lambda_{v} \sum_{w \in R_{\Gamma(\pi)}(v)} p_{v, w} w$, and $p_{v, w} \geq \min \left(\frac{1}{2}, \frac{\varepsilon}{2}\right)$.

Proof. Let $r$ and $s$ denote the source and target regions. Let $\Omega=\min \left(\frac{\varepsilon}{2}, \frac{1}{2}\right)$.

Assume that both $\inf (s)$ and $\sup (s)$ have indegree 1 , and let $u$ be a predecessor. We have,

$$
\begin{aligned}
& \lambda_{\inf (s)}^{\prime}=\lambda_{u} \cdot p_{u, \inf (s)}, \\
& \lambda_{\sup (s)}^{\prime}=\lambda_{u} \cdot p_{u, \sup (s)} .
\end{aligned}
$$

If $u$ is the only vertex of $r$, then $\lambda_{u}=1$, and we get that $p_{u, \inf (s)}, p_{u, \sup (s)} \geq \varepsilon$ since $\lambda_{\inf (s)}^{\prime}, \lambda_{\sup (s)}^{\prime} \geq \varepsilon$. Otherwise, we must have $\lambda_{u} \leq 1-\varepsilon$. Again, because $\lambda_{\inf (s)}^{\prime}, \lambda_{\text {sup }(s)}^{\prime} \geq \varepsilon$, we get that $p_{u, \inf (s)}, p_{u, \sup (s)} \geq \frac{\varepsilon}{1-\varepsilon} \geq \Omega$. All other edges have outdegree 1 so the probabilities are equal to 1 .

Assume now that $\sup (s)$ has indegree 2 , but $\inf (s)$ has indegree 1 . We show that in this case $r=s$. Observe that $\inf (s)$ cannot have indegree higher than that of $\sup (s)$ since any predecessor of $\inf (s)$ is a predecessor of $\sup (s)$. Now, $\sup (s)$ cannot have both its predecessors outside $\mathcal{V}(s)$ since otherwise these would be predecessors of $\inf (s)$. Thus, $\inf (s) \in \mathcal{V}(r)$, and is a predecessor of $\sup (s)$. In this case, $\inf (s)$ is a predecessor of itself since this is a delay transition. Furthermore, if $\sup (s)$ has its other predecessor outside $s$, this would again be a predecesor of $\inf (s)$, and $\inf (s)$ would have indegree 2 . Therefore, the predecessors of $\sup (s)$ must be $\sup (s)$ and $\inf (s)$, and the predecessor of $\inf (s)$ is itself. This is only possible when $s=r$.

We have,

$$
\begin{aligned}
& \lambda_{\inf (s)}^{\prime}=\lambda_{\inf (s)} \cdot p_{\inf (s), \inf (s)}, \\
& \lambda_{\sup (s)}^{\prime}=\lambda_{\inf (s)} \cdot p_{\inf (s), \sup (s)}+\lambda_{\sup (s)} \cdot p_{\sup (s), \sup (s)} .
\end{aligned}
$$

We get that $p_{\inf (s), \inf (s)} \geq \Omega$. Here, $p_{\sup (s), \sup (s)}=1 \operatorname{since} \sup (s)$ has outdegree 1 by assumption. Let $x$ denote the clock with the least fractional value in $s$. Notice that the clock $x$ has the same value in all vertices apart from $\sup (s)$. We have $\nu(x)=\inf (s)(x) \cdot\left(1-\lambda_{\sup (s)}\right)+\sup (s)(x) \cdot \lambda_{\sup (s)}$, which gives $\nu(x)-\inf (s)(x)=$ $\lambda_{\sup (s)}$. Similarly, we have $\nu^{\prime}(x)-\inf (s)(x)=\lambda_{\sup (s)}^{\prime}$. Since the delay is at least $\varepsilon$, we have

$$
\left(\nu^{\prime}(x)-\inf (s)(x)\right)-(\nu(x)-\inf (s)(x))=\nu^{\prime}(x)-\nu(x) \geq \varepsilon .
$$

which is equivalent to $\lambda_{\sup (s)}^{\prime}-\lambda_{\sup (s)} \geq \varepsilon$. By writing $\lambda_{\sup (s)}^{\prime}$ as above, this is equivalent to

$$
\lambda_{\inf (s)} \cdot p_{\inf (s), \sup (s)}+\lambda_{\sup (s)} \cdot\left(p_{\sup (s), \sup (s)}-1\right) \geq \varepsilon .
$$


Thus, we must have $\lambda_{\inf (s)} \cdot p_{\inf (s), \sup (s)} \geq \varepsilon$, which means that $p_{\inf (s), \sup (s)} \geq$ $\frac{\varepsilon}{1-\varepsilon} \geq \varepsilon$, as required.

Let us now consider the case where two vertices of $r$ have outdegree 2 . This means that $\inf (r)$ and $\sup (r)$ are different, and that they have an edge leading to both $\inf (s)$ and $\sup (s)$. To simplify notations, let us write $r_{1}=\inf (r), r_{2}=\sup (r)$ and similarly $s_{1}$ and $s_{2}$ for $s$. We have the following relation.

$$
\begin{aligned}
& \lambda_{s_{1}}^{\prime}=p_{11} \lambda_{r_{1}}+p_{21} \lambda_{r_{2}}, \\
& \lambda_{s_{2}}^{\prime}=p_{12} \lambda_{r_{1}}+p_{22} \lambda_{r_{2}},
\end{aligned}
$$

where $p_{11}, p_{12}, p_{21}, p_{22} \geq 0$ are given by Lemma 5 and satisfy $p_{11}+p_{12}=1$, $p_{21}+p_{22}=1$. Now, all $p_{i j}$ may not be greater than $\Omega$ here. But we will show that one can then modify these to obtain probabilities at least $\Omega$, and still satisfy the above equation. Without loss of generality, let us assume that $p_{11}<\Omega$. We would like to increase $p_{11}$ by some $\alpha \geq 0$ and still satisfy the above equation, the stochasticity (i.e. $p_{i 1}+p_{i 2}=1$ ), and obtain a new set of probabilities $p_{i j}$ whose all components are in $[\Omega, 1-\Omega]$. The modified equation is the following:

$$
\begin{aligned}
& \lambda_{s_{1}}^{\prime}=\left(p_{11}+\alpha\right) \lambda_{r_{1}}+\left(p_{21}-\frac{\alpha \lambda_{r_{1}}}{\lambda_{r_{2}}}\right) \lambda_{r_{2}}, \\
& \lambda_{s_{2}}^{\prime}=\left(p_{12}-\alpha\right) \lambda_{r_{1}}+\left(p_{22}+\frac{\alpha \lambda_{r_{1}}}{\lambda_{r_{2}}}\right) \lambda_{r_{2}} .
\end{aligned}
$$

This equation is satisfied for any $\alpha$, assuming (1) is satisfied. In order to obtain the matrix we are looking for, it suffices to find $\alpha$ such that the following constraints are satisfied (since stochasticity is already satisfied).

$$
\begin{gathered}
\Omega \leq p_{11}+\alpha \leq 1-\Omega, \\
\Omega \leq p_{21}-\frac{\alpha \lambda_{r_{1}}}{\lambda_{r_{2}}} \leq 1-\Omega .
\end{gathered}
$$

We now use Fourier-Motzkin elimination to show that there is always such a choice for $\alpha$. Let us rewrite the above constraints on $\alpha$ by separating lower bounds and upper bounds.

$$
\begin{array}{r}
\Omega-p_{11} \leq \alpha, \\
\frac{\lambda_{r_{2}}}{\lambda_{r_{1}}} p_{21}+\frac{\lambda_{r_{2}}}{\lambda_{r_{1}}}(1+\Omega) \leq \alpha, \\
\alpha \leq 1-\Omega-p_{11}, \\
\alpha \leq p_{21} \frac{\lambda_{r_{2}}}{\lambda_{r_{1}}}-\Omega \frac{\lambda_{r_{2}}}{\lambda_{r_{1}}} .
\end{array}
$$

It suffices to show that each pair of lower and upper bounds is satisfied by some $\alpha$.

(4)-(6): holds since $\Omega<\frac{1}{2}$.

(4)-(7): This can be written as $\Omega \leq \frac{\lambda_{r_{2}}}{\lambda_{r_{1}}+\lambda_{r_{2}}} p_{21}+\frac{\lambda_{r_{1}}}{\lambda_{r_{1}}+\lambda_{r_{2}}} p_{11}$. But $\varepsilon \leq$ $\lambda_{r_{2}} p_{21}+\lambda_{r_{1}} p_{1} 1$ since this is equal to $\lambda_{s_{1}}^{\prime}$. Since $\lambda_{r_{1}}+\lambda_{r_{2}} \leq 1$ and $\Omega \leq \varepsilon$, the constraint holds. 
(5)-(6): This is equivalent to

$$
\begin{aligned}
\Omega & \leq \frac{\lambda_{r_{1}}}{\lambda_{r_{1}}+\lambda_{r_{2}}}\left(1-p_{11}\right)+\frac{\lambda_{r_{2}}}{\lambda_{r_{1}}+\lambda_{r_{2}}}\left(1-p_{21}\right) \\
& =1-\left(\frac{\lambda_{r_{1}}}{\lambda_{r_{1}}+\lambda_{r_{2}}} p_{11}+\frac{\lambda_{r_{2}}}{\lambda_{r_{1}}+\lambda_{r_{2}}} p_{21}\right) .
\end{aligned}
$$

We have $\frac{\lambda_{r_{1}}}{\lambda_{r_{1}}+\lambda_{r_{2}}} p_{11}+\frac{\lambda_{r_{2}}}{\lambda_{r_{1}}+\lambda_{r_{2}}} p_{21} \leq \frac{\lambda_{r_{1}}}{\lambda_{r_{1}}+\lambda_{r_{2}}} \Omega+\frac{\lambda_{r_{2}}}{\lambda_{r_{1}}+\lambda_{r_{2}}}$ since $p_{11}<\Omega$ and $p_{21} \leq 1$. So, it suffices to show that $\Omega \leq 1-\frac{\lambda_{r_{1}}}{\lambda_{r_{1}}+\lambda_{r_{2}}} \Omega-\frac{\lambda_{r_{1}}}{\lambda_{r_{1}}+\lambda_{r_{2}}}$, i.e. $\Omega \leq \frac{\lambda_{r_{1}}}{2 \lambda_{r_{1}}+\lambda_{r_{2}}}$. But this holds since $\varepsilon \leq \lambda_{r_{1}}$ and $2 \lambda_{r_{1}}+\lambda_{r_{2}} \leq 2-\varepsilon$. In fact, we have $\Omega \leq \frac{\varepsilon}{2-\varepsilon}$.

(5)-(7): This is equivalent to $2 \frac{\lambda_{r_{2}}}{\lambda_{r_{1}}} \Omega \leq \frac{\lambda_{r_{2}}}{\lambda_{r_{1}}}$ and holds since $\Omega \leq \frac{1}{2}$.

Decreasing Lyapunov function We note the following property of the functions $L_{I}(\cdot)$, proved by induction using Lemma 5 .

Lemma 20. Let $\pi$ be a cycle, and write $\gamma(\pi)=\left(V_{1} \cup \ldots \cup V_{n}, f_{G}, E\right)$ for its orbit graph, and $\Gamma(\pi)=\left(V, f_{G}^{\prime}, E^{\prime}\right)$ for its folded orbit graph. Let $I_{1} \subseteq V$ be any subset for which $(u, v) \in E^{\prime}$ with $v \in I_{1}$ implies $u \in I_{1}$. Define $I_{i}=\operatorname{Succ}_{\gamma(\pi)}\left(I_{i-1}\right)$ for all $2 \leq i \leq n$. Then, for any run $\rho$ along $\pi$, we have $L_{I_{i}}\left(\operatorname{state}_{i}(\rho)\right)=L_{I_{1}}\left(\operatorname{state}_{1}(\rho)\right)$ for all $1 \leq i \leq n$.

Lemma 7 . Let $\pi$ be a non-punctual progress cycle, and $(\ell, \nu) \stackrel{\pi}{\rightarrow}\left(\ell, \nu^{\prime}\right)$ a run along $\pi$. Then, there exists $\varepsilon>0$ such that there exists a run from $(\ell, \nu)$, along $\pi$, to any point in $\{\ell\} \times\left(\right.$ Ball $\left._{d_{\infty}}\left(\nu^{\prime}, \varepsilon\right) \cap\left[\nu^{\prime}\right]\right)$.

Proof. Consider the partition of the clocks inside first $(\pi)$, according to their fractional ordering. One can factor $\pi$ as $\pi=\pi^{\prime} \pi_{n} \pi_{n-1} \ldots \pi_{1}$, where each $\pi_{i}$ starts with the latest reset of some clocks $Y_{i} \subseteq \mathcal{C}$, with $Y_{i}=X_{j}$ for some $j$, and $\pi_{1}$ ends with a reset of the clocks $X_{0}$ if this set is non-empty. To see that for any $i$, there exists $j$ such that $Y_{i}=X_{j}$, suppose the latest resets of some clocks $x$ and $y$ are at different transitions, say, $\operatorname{state}_{i}(\pi)$ and state $_{j}(\pi)$ respectively, with $i<j$. Then, they belong to the same set $X_{k}$ if, and only if the total delay of any run along $\pi_{i \ldots j}$ is an integer. But this is only possible if equality constraints are used, which is not the case since $\pi$ is non-punctual. Moreover, we have $n=m$, since all clocks are reset at least once as $\pi$ is a progress cycle.

Let us factorize the run $\rho$ as $\rho=\rho^{\prime} \rho_{m} \rho_{m-1} \ldots \rho_{1}$ where each $\rho_{i}$ is along $\pi_{i}$ and $\rho^{\prime}$ along $\pi^{\prime}$. Then, for each clock $x \in X_{i}, \nu^{\prime}(x)=\operatorname{delay}\left(\rho_{i} \rho_{i-1} \ldots \rho_{1}\right)$, where delay $(\cdot)$ denotes the sum of the delays of a given run. Consider the timed trace $\left(d_{i}, e_{i}\right)_{i}$ of $\rho$. We have $d_{i}>0$ for all $i$ by definition. By Proposition 3 , any timed trace $\left(d_{i}^{\prime}, e_{i}\right)_{i}$, with $\left|d_{i}-d_{i}^{\prime}\right| \leq \varepsilon$ for all $i \geq 1$, defines a run starting at $(\ell, \nu)$ along $\pi$, if $\varepsilon$ is chosen small enough. It remains to show that any point $\nu^{\prime \prime} \in$ Ball $_{d_{\infty}}\left(\nu^{\prime}, \varepsilon\right) \cap\left[\nu^{\prime}\right]$ is reachable from $(\ell, \nu)$ following such a "close" timed trace.

Consider such an $\varepsilon$, and any $\nu^{\prime \prime} \in\left[\nu^{\prime}\right]$ such that $d_{\infty}\left(\nu^{\prime}, \nu^{\prime \prime}\right) \leq \frac{\varepsilon}{2}$. Notice that we have $\operatorname{frac}\left(\nu^{\prime \prime}(x)\right)=\operatorname{frac}\left(\nu^{\prime \prime}(y)\right)$, and $\operatorname{frac}\left(\nu^{\prime}(x)\right)=\operatorname{frac}\left(\nu^{\prime}(y)\right)$ for any $x, y \in Y_{i}$, which implies $\nu^{\prime \prime}(x)-\nu^{\prime}(x)=\nu^{\prime \prime}(y)-\nu^{\prime}(y)$. Thus, we define $\left(\eta_{1}, \ldots, \eta_{m}\right) \in\left[-\frac{\varepsilon}{2}, \frac{\varepsilon}{2}\right]^{m}$ such that $\nu^{\prime}(x)+\eta_{i}=\nu^{\prime \prime}(x)$ for all $x \in Y_{i}$. A run ending 
in $\left(\ell, \nu^{\prime \prime}\right)$ can be defined as $o=o^{\prime} o_{m} \ldots o_{1}$, by modifying the trace $\left(d_{i}, e_{i}\right)_{i}$ so as to satisfy the following properties:

$$
\begin{aligned}
& \text { delay }\left(o_{1}\right)=\operatorname{delay}\left(\rho_{1}\right)+\eta_{1}, \\
& \operatorname{delay}\left(o_{2}\right)=\operatorname{delay}\left(\rho_{2}\right)+\eta_{2}-\eta_{1}, \\
& \ldots \\
& \text { delay }\left(o_{m}\right)=\operatorname{delay}\left(\rho_{m}\right)+\eta_{m}-\eta_{m-1}, \\
& \text { delay }\left(o^{\prime}\right)=\operatorname{delay}\left(\rho^{\prime}\right)
\end{aligned}
$$

It is possible to obtain $o$ by modifying an arbitrary delay in each $\rho_{i}$ since $\left|\eta_{i}-\eta_{i+1}\right| \leq \varepsilon$.

Lemma 8. The folded orbit graph of any non-punctual progress cycle is connected.

Proof. Consider a non-punctual progress cycle and let us denote $r=\operatorname{first}(\pi)$. Consider the basis $\mathcal{B}_{v_{0}}$, with $v_{0}=\inf (r)$, defined in Lemma 2. Assume that the orbit graph has at least two disjoint connected components $G_{1}$ and $G_{2}$. Note that we do not require $G_{i}$ 's to be strongly connected components. These are simply two disjoint maximal subgraphs. Let $v_{0}+u$ be a point in $r$, with $u \in \operatorname{Span}\left(\mathcal{B}_{v_{0}}\right)$ and write $u=\sum_{v} \lambda_{v} v$. Let $f$ denote the linear function that associates to each element of $\operatorname{Span}\left(\mathcal{B}_{v_{0}}\right)$ the sum of the coefficients of the vertices of $G_{1}$. Then $f(u)=\sum_{v \in G_{1}} \lambda_{v}$. For any run $\left(v_{0}+u\right) \stackrel{\pi}{\rightarrow}\left(v_{0}+u^{\prime}\right)$, where $u^{\prime}=\sum_{v} \lambda_{v}^{\prime} v$, we have, by Lemma $20, f(u)=f\left(u^{\prime}\right)$. Thus, the set $U \subseteq r$ of valuations reachable from $v_{0}+u$ along $\pi$ lies in the set $v_{0}+f^{-1}(f(u))$, which is an affine hyperplane of $v_{0}+\operatorname{Span}\left(\mathcal{B}_{v_{0}}\right)$. So it has smaller dimension than $r$. On the other hand, by Lemma 7, the set $U$ includes the intersection of a $d$-open ball intersected with $r$, hence have the same dimension as $r$. Contradiction.

Lemma 9. Let $\omega \in$ Outcome $_{\mathcal{A}}^{\delta}\left(\cdot, \sigma_{\delta}^{P}\right)$, and $\rho$ be a finite prefix of $\omega$ such that $\pi$, the projection of $\rho$ to regions, is a cycle. If $\pi$ is a non-forgetful progress cycle, then, writing first $(\rho)=\boldsymbol{\lambda} \boldsymbol{v}$ and last $(\rho)=\boldsymbol{\lambda}^{\prime} \boldsymbol{v}^{\prime}$, we have, $\sum_{i \in I(\pi)} \lambda_{i}^{\prime} \leq \sum_{i \in I(\pi)} \lambda_{i}-\varepsilon^{2} / 2$.

Proof. We know that $\pi$ is non-punctual by definition of $\sigma_{\delta}^{\mathrm{P}}$. Let us write $\gamma(\pi)=$ $\left(V_{1} \cup \ldots \cup V_{n}, f_{G}, E\right)$. For each $1 \leq i \leq n$, let $I_{i}$ denote the set of nodes of $V_{i}$ that are reachable from the nodes $\{1\} \times I(\pi) \subseteq V_{1}$. Observe that $I_{1}=\{1\} \times I(\pi)$, and $\{n\} \times I(\pi) \subseteq I_{n}$ since $I(\pi)$ is a strongly connected component. However, $I_{n} \neq\{n\} \times I(\pi)$ because of Corollary 1. Let $J_{i}$ denote the set of nodes of $V_{i}$ from which the set $\{n\} \times I(\pi)$ is reachable. We have $J_{n}=\{n\} \times I(\pi)$ by definition. Because $I(\pi)$ is an initial component, we have $J_{i} \subseteq I_{i}$ for all $i$. Indeed, assume $v \in J_{i} \backslash I_{i}$ for some $i$. Then some node of $\{n\} \times I(\pi)$ is reachable from $v$. Since each node has at least one predecessor in $\gamma(\pi), v$ is reachable from some node $u$ of $V_{1}$. We have that $u \notin I_{1}$, since otherwise $v \in I_{i}$. Thus, in $\Gamma(\pi)$, there is an edge from the SCC of $u$ to $I(\pi)$, contradicting the fact that $I(\pi)$ is an initial SCC. Moreover, we have $I_{1}=J_{1}$. In fact, $J_{n}$ is reachable from any node of $I_{1}$ since this is a strongly connected component.

Let $i_{0}$ be the least index such that $I_{i} \neq J_{i}$. Notice that we have $L_{I_{i}}\left(\right.$ state $\left._{i}(\rho)\right)=$ $L_{I_{1}}\left(\right.$ state $\left._{1}(\rho)\right)$ for any $1 \leq i \leq i_{0}-1$, by Lemma 5 (in fact, all predecessors of $I_{i}$ are 
in $I_{i-1}$, and all nodes of $I_{i-1}$ have at least one successor in $\left.I_{i}\right)$. Moreover, for any $1 \leq i \leq n$, we have $L_{J_{i}}$ ( state $\left._{i}(\rho)\right) \geq L_{J_{n}}\left(\right.$ state $\left._{n}(\rho)\right)$, since all predecessors of $J_{i}$ are in $J_{i-1}$ by definition. We will show that $L_{J_{i_{0}}}\left(\right.$ state $\left._{i_{0}}(\rho)\right) \leq L_{J_{i_{0}-1}}\left(\right.$ state $\left._{i_{0}-1}(\rho)\right)-$ $\varepsilon^{2} / 2$, which thus implies the desired inequality.

There exists $a \in J_{i_{0}-1}$ and $b \in I_{i_{0}} \backslash J_{i_{0}}$ with $(a, b) \in E$. Let us write $\boldsymbol{\lambda} \boldsymbol{v}=$ state $_{i_{0}-1}(\rho)$. By Proposition 1 , we have $\lambda_{a} \geq \varepsilon$. Since $a \in J_{i_{0}-1}$ and $b \notin J_{i_{0}}, a$ must have another successor in $\gamma(\pi)$. Hence, the transition from state $i_{0}-1$ to $i_{0}$ must be a delay (otherwise each vertex has a unique successor). By Proposition 2 , the edge $(a, b)$ receives a probability of at least $\varepsilon / 2$ (in Lemma 5 ). The inequality follows.

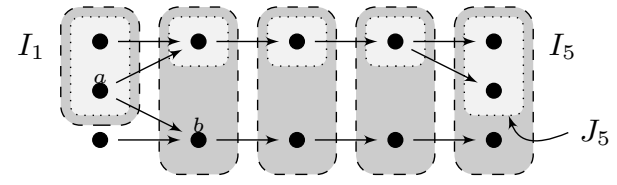

Fig. 8. Sets $I_{i}$ and $J_{i}$ for the cycle of Fig. 2

We introduce some notations to prove Lemma 10.

A graph $G=(V, E)$ is a linear-order-graph if there exists a linear order $\prec$ on $V$ such that $(i, j) \in E$ if, and only if $i \prec j$ for all $i, j \in V$. An $r$-coloring is a function $f: E \rightarrow\{1, \ldots, r\}$. A monochromatic path is a path in which all edges have the same color.

Theorem 21 ([San11]). Let $G=(V, E)$ be a linear-order-graph over $n$ nodes given with an $r$-coloring. Then $G$ contains a monochromatic directed path of length $\lfloor\sqrt{n / r-2}\rfloor-1$.

Note that Ramsey's theorem would actually suffice for our proof but it would give an exponentially larger bound than Theorem 21. Consequently, the proof below will bound by a simple exponential the number of steps to the "failure" under any strategy in non-robust systems.

Lemma 10. Let $\pi$ be a path of $\mathcal{R}(\mathcal{A})$ written as $\pi=\pi_{0} \pi_{1} \pi_{2} \ldots \pi_{n}$ where each $\pi_{i}$ is a cycle that starts in the same state, for $i \geq 1$. Then, one can write $\pi=\pi_{0}^{\prime} \pi_{1}^{\prime} \pi_{2}^{\prime} \ldots \pi_{m+1}^{\prime}$ such that $m \geq \sqrt{n / r-2}-1$, where $r=2^{(|\mathcal{C}|+1)^{2}}|\mathcal{R}(\mathcal{A})|$, and for some indices $0=\alpha_{0}<\alpha_{1}<\ldots$, we have $\pi_{i}^{\prime}=\pi_{\alpha_{i}} \cdot \ldots \cdot \pi_{\alpha_{i+1}-1}$ for each $i \geq 0$, and $\Gamma\left(\pi_{1}^{\prime}\right)=\Gamma\left(\pi_{i}^{\prime}\right)$ for all $1 \leq i \leq m$.

Proof. We will apply this theorem as follows. Given a cycle $\pi$ factorized as $\pi=$ $\pi_{1} \pi_{2} \ldots \pi_{n}$, in which all $\pi_{i}$ are cycles from the same region-states, we consider a linear-order-graph $G=(V, E)$ with $V=\{1, \ldots, n\}$ and $E$ is defined by the usual order on integers. The coloring function $f$ is given as follows. For all $i<j$, we define $f((i, j))=\Gamma\left(\pi_{i} \pi_{i+1} \ldots \pi_{j}\right)$. Thus, a monochromatic path given by previous theorem corresponds to a factorization into $\pi=\pi_{0} \cdot\left(\pi_{\alpha_{1}} \ldots \pi_{\alpha_{2}-1}\right)$. $\left(\pi_{\alpha_{2}} \ldots \pi_{\alpha_{3}-1}\right) \cdot \ldots$ such that all $\Gamma\left(\pi_{\alpha_{j}} \ldots \pi_{\alpha_{j+1}-1}\right)$ define the same graph. 


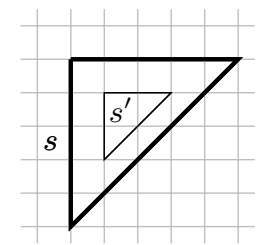

Fig. 9. A subregion $s^{\prime}$ of $s$ with smaller granularity

\section{B.4 Proofs of Section 6}

\section{Controllable Predecessors}

Lemma 12. Let $\pi$ be a non-punctual path from region $r$ to $s$. Let $s^{\prime} \subseteq s$ such that there exists $\nu^{\prime} \in s^{\prime}$ and $\varepsilon>0$ with Ball $d_{\infty}\left(\nu^{\prime}, \varepsilon\right) \cap s \subseteq s^{\prime}$. Then, $\operatorname{CPre}_{\pi}^{\delta}\left(s^{\prime}\right)$ is non-empty for small enough $\delta>0$.

Proof. Note that the requirement on $s^{\prime}$ says that $s^{\prime}$ should have non-empty interior in the $d_{\infty}$-topology induced on the set $s$. Consider such a set $s^{\prime}$ and a valuation $\nu^{\prime} \in$ $s^{\prime}$ with $B=\operatorname{Ball}_{d_{\infty}}\left(\nu^{\prime}, \varepsilon\right) \cap s \subseteq s^{\prime}$ for some $\varepsilon>0$. Let $\rho$ be any run along $\pi$ from some $\nu \in r$ to $\nu^{\prime}$, and let $u=\left(d_{i}, e_{i}\right)_{1 \leq i \leq n}$ denote the timed trace of $\rho$. Choose $0<$ $\varepsilon^{\prime}<\varepsilon$ so that all timed traces in $\operatorname{Ball}_{d}\left(u, \varepsilon^{\prime}\right)$ are feasible, and lead into $B$. This is possible since all delays are positive, by Prop. 3 ( $\pi$ is non-punctual), and by the fact that the value of each clock at the last state of a run is continuously determined by the delays in the timed trace. Let $\delta \leq \varepsilon^{\prime}$. Now, starting at $\nu$, if Controller plays according to $u$, then the timed trace of any outcome belongs to $\operatorname{Ball}_{d}\left(u, \varepsilon^{\prime}\right)$, thus is feasible and ends in $B$. More precisely, Controller's strategy that ensures reaching $B$ consists simply in playing $u$ from $\nu$. Therefore, $\nu \in \operatorname{CPre}_{\pi}^{\delta}\left(s^{\prime}\right)$.

\section{Winning Under Perturbations}

Lemma 13. Let $\pi$ be an aperiodic cycle. Then, there exists $n \leq\left|\mathcal{C}_{0}\right| \cdot\left|\mathcal{C}_{0}\right|$ ! such that $\Gamma\left(\pi^{n}\right)$ is a complete graph.

Proof. For any vertex $v$, there exists $n_{v} \leq|V|$ such that $(v, v) \in \Gamma\left(\pi^{n_{v}}\right)$ since $\Gamma(\pi)$ is strongly connected. Let $m=\operatorname{Icm}_{v \in V}\left(n_{v}\right)$. Then, $(v, v) \in \Gamma\left(\pi^{m}\right)$ for any vertex $v \in V$. Note that $m \leq|V|$ !. Let us write $\pi^{\prime}=\pi^{m}$. Consider the length $n_{v, w}$ of the shortest path from $v$ to $w$ in $\Gamma\left(\pi^{\prime}\right)$ for any $v, w \in V$, and let $m^{\prime}=\max _{v, w \in V} n_{v, w}$, which is finite since $\pi^{\prime}$ is strongly connected. We have, $(v, w) \in \Gamma\left({\pi^{\prime}}^{\prime}\right)$. In fact, $(v, v) \in \Gamma\left(\pi^{\prime m^{\prime}-n_{v, w}}\right)$ since $(v, v) \in \Gamma\left(\pi^{\prime}\right)$ and $(v, w) \in \Gamma\left(\pi^{\prime n_{v, w}}\right)$. We have, $m^{\prime} \leq|V|$, so $n$ can be chosen as $|V| \cdot|V| !$.

Lemma 14. For any non-empty $D B M M$, there exists a non-empty $D B M N$ such that Ball $d_{\infty}(\nu, \varepsilon) \cap M \subseteq N$ for some $\nu \in M$, and for any shrinking matrix $P$ with $(M, P) \neq \emptyset, N \subseteq(M, P)$. Moreover, $N$ is computable in polynomial time. 
Proof. We define $Q$ as follows. For any $x, y \in \mathcal{C}_{0}$, if $M_{x, y}=-M_{y, x}$, then $Q_{x, y}=$ $Q_{y, x}=0$. Otherwise, we have $\mathrm{M}_{x, y} \geq-\mathrm{M}_{x, y}+1$, and in this case, we let $Q_{x, y}=$ $Q_{y, x}=1$. Let us show that $M-\varepsilon Q \neq \emptyset$, for small enough $\varepsilon>0$. To show nonemptiness, it suffices to show that the constraints of the DBM $M-\varepsilon Q$ do not imply a negative upper bound on zero [BY04]. For a contradiction, assume there is a sequence of indices $x_{1}, x_{2}, \ldots, x_{n} \in \mathcal{C}_{0}$, with $x_{1}=x_{n}$, such that $\sum_{i=0}^{n-1}\left(M_{x_{i}, x_{i+1}}-\right.$ $\left.\varepsilon Q_{x_{i}, x_{i+1}}\right)<(0, \leq)$ for all $\varepsilon>0$. This means that $\sum_{i=0}^{n-1} M_{x_{i}, x_{i+1}} \leq(0, \leq)$, but since $M$ is non-empty, we have $\sum_{i=0}^{n-1} M_{x_{i}, x_{i+1}}=(0, \leq)$. But then, for all $1 \leq i \leq n-1$, we must have $M_{x_{i}, x_{i+1}}=-M_{x_{i+1}, x_{i}}$, which implies $Q_{x_{i}, x_{i+1}}=0$ by definition, thus we cannot have $\sum_{i=0}^{n-1}\left(M_{x_{i}, x_{i+1}}-\varepsilon Q_{x_{i}, x_{i+1}}\right)<(0, \leq)$. To see that $M_{x_{i}, x_{i+1}}=-M_{x_{i+1}, x_{i}}$, observe that if $-M_{x_{i+1}, x_{i}}<M_{x_{i}, x_{i+1}}$ for some $i$, then, for any $\alpha \in(0,1)$, there exists a valuation $\nu$ in $M$, and satisfying $\nu\left(x_{i}\right)-\nu\left(x_{i+1}\right)=$ $M_{x_{i}, x_{i+1}}-\alpha$. But since $\nu\left(x_{j}\right)-\nu\left(x_{j+1}\right) \leq M_{x_{j}, x_{j+1}}$ for all $1 \leq j \leq n$, this implies that $\nu\left(x_{1}\right)-\nu\left(x_{2}\right)+\nu\left(x_{2}\right)-\nu\left(x_{3}\right)+\ldots-\nu\left(x_{n}\right)<\sum_{j=1}^{n-1} M_{x_{j}, x_{j+1}}-\nu<(0, \leq)$, which is a contradiction.

Now, we choose $\varepsilon$ as above, and small enough so that $M_{x, y} \neq-M_{y, x}$ implies that $M_{x, y}-\varepsilon Q_{x, y}>-M_{y, x}+\varepsilon Q_{y, x}$. We let $N=M-\varepsilon Q$. Then, given any shrunk DBM $(M, P)$, we have $M_{x, y}-\varepsilon Q_{x, y}<M_{x, y}-\delta P_{x, y}$, for small enough $\delta>0$ as required. Since $\varepsilon$ can be chosen arbitrarily small, $N$ also has non-empty interior in the topology induced on $M$.

Note that $N$ defined here could be given by scaling $r$, i.e. applying a homothety as in Figure 9. 